\title{
Numerical Buoyancy-Wave Model for Wave Stress and Drag Simulations in the Atmosphere
}

\author{
M. Zirk*, R. Rõõm, A. Männik, A. Luhamaa, M. Kaasik and \\ S. Traud \\ Institute of Physics, Tartu University, Tartu 50090, Estonia.
}

Received 28 November 2012; Accepted (in revised version) 8 May 2013

Communicated by Lianjie Huang

Available online 15 August 2013

\begin{abstract}
Orographic drag formation is investigated using a numerical wave model (NWM), based on the pressure-coordinate dynamics of non-hydrostatic HIRLAM. The surface drag, wave stress (vertical flux of horizontal momentum), and wave drag are split to the longitudinal and transverse components and presented as Fourier sums of their spectral amplitudes weighted with the power spectrum of relative orographic height. The NWM is accomplished, enabling a spectral investigation of the buoyancy wave stress, and drag generation by orography and is then applied to a cold front, characterised by low static stability of the upper troposphere, large vertical and directional wind variations, and intensive trapped wave generation downstream of obstacles. Resonances are discovered in the stress and drag spectra in the form of high narrow peaks. The stress conservation problem is revisited. Longitudinal stress conserves in unidirectional flow, 2D orography conditions, but becomes convergent for rotating wind or 3D orography. Even in the convergent case the vertical momentum flux from the troposphere to stratosphere remains substantial. The transverse stress never conserves. Disappearing at the surface and on the top, it realises the main momentum exchange between lower an upper parts of the troposphere. Existence of stationary stratospheric quasi-turbulence (SQT) is established above wind minimum in the stratosphere.
\end{abstract}

PACS: $92.60 . K c, 92.70 . \mathrm{Bc}, 92.60 . \mathrm{hh}, 02.30 . \mathrm{Nw}$, 47.11.Kb, 47.27.er

Key words: Fourier spectrum, orographic buoyancy waves, surface drag, wave drag, wave stress, momentum flux, wave equation, numerical wave modelling.

${ }^{*}$ Corresponding author. Email addresses: Marko.Zirk@ut.ee (M. Zirk), rein.room@ut.ee (R. Rõõm), Aarne.Mannik@ut.ee (A. Männik), Andres.Luhamaa@ut.ee (A. Luhamaa), Marko.Kaasik@ut.ee (M. Kaasik), Sebastian. Traud@ut.ee (S. Traud) 


\section{Introduction}

In recent years, wave and wave-originated drag modelling have become vital for applications of drag parameterisation in global circulation, numerical weather prediction, and climate studies [1-11]. Among different buoyancy waves, orographic waves are the most important drag source. The two kinds of drag that affect laminar atmospheric flow by terrain are surface drag and wave drag. Surface drag was first introduced by [12] and subsequently discussed in early studies by $[13,14]$, and for three-dimensional orography, by [15]. It affects the near-surface momentum mainly (as it will be demonstrated hereafter, the surface drag will vanish above mountain crests, producing wind weakening in the front and the lees of mountains and yielding the blocking and envelope-orography formation). Wave drag is caused by the wave stress (which is the same as the waveoriginated vertical flux of horizontal momentum) convergence in the upper-level turbulent layers, which are created by the same waves when they break. Such an upper-level drag mechanism was discussed in the pioneering work by [16] (see also [17, 18]), who showed that wave stress is conserved in mean zonal flow (now known as the EliassenPalm momentum flux conservation theorem), and proposed the wave breaking with following turbulent dissipation as a probable mechanism, capable of the upper-level momentum change explanation. As in this paper will be demonstrated, the major part of the stress will pass from the troposphere to the stratosphere without notable conversion even if it is not strictly conserved.

Whereas upper-level wave-breaking induced turbulence still remains beyond the capabilities of numerical weather prediction and climate models and requires sub-grid parameterisation, generation of surface drag and wave stress can be modelled numerically because contemporary non-hydrostatic models do provide the necessary resolution. The most straightforward method for stress and drag studies lies in modelling wave generation by orography. There exist a great number of research papers dedicated to wave generation by orography and wave-related drag problems. In some of the most cited works, the problem has been studied both numerically by integrating non-stationary, non-linear, and typically non-hydrostatic equations of atmospheric dynamics [19-29], and analytically by solving linear wave equations [30-38].

In the present paper, we will revisit the orographic drag generation problem using an approach for wave and wave-related drag study that combines the power of the linear spectral method with the flexibility of numerical approach. A previously developed numerical solution method [39], RZ07 hereafter, for linear spectral wave equation is extended to include stress and drag computation in a general non-stationary case for any arbitrary thermal and wind stratification. The updated numerical wave model (NWM) is then applied to wave and accompanying stress and drag modelling in the real atmosphere. Using a friction-free, non-reflective (radiative) upper boundary condition, NWM can handle waves vertically up to the mesosphere. The departure wave equation in RZ07 is based on the Miller-Pearce [40,41] anelastic, non-hydrostatic, pressure-coordinate equations, previously implemented in non-hydrostatic HIRLAM [42, 43]. These equa- 
tions are three-dimensionally non-divergent in isobaric coordinates and hence filter acoustic disturbances; they are omni-spectral, i.e., can describe waves from the planetary scale to boundary-layer micro-waves without phase distortions [44].

The main problem with drag implementation in an NWM framework is that the stress and drag vectors are initially defined in common Cartesian coordinates, whereas the wave equation solution, which is the pressure vertical velocity (the omega velocity or $\omega$ hereafter) intended to be used for evaluating these vectors, is presented vertically in pressure coordinates and horizontally in the form of normal (Fourier) modes in wavevector space. Thus, for drag and stress evaluation from $\omega$, their horizontally spectral, vertically pressure-coordinate presentations are required. Due to the lack of such tools previously, we developed the necessary formalism from scratch. In Sections 2 and 3, a concise, wave-originated spectral pressure-coordinate drag theory is developed. In Section 2, departing from the Miller-Pearce equations, the drag and stress vectors are introduced via a horizontal momentum balance equation in pressure coordinates. The surface drag, wave stress, and wave drag are expressed via basic dynamic fields. The stress and drag vectors are initially decomposed to potential and rotational parts, which are named the longitudinal and transverse stress and drag because their normal-mode amplitudes appear to be opposite and transverse, respectively, to the wave vector. Such decomposition proceeds from the splitting of horizontal wind and dynamic forcing to the potential and rotational constituents. In turn, the wind splitting is needed for the closed formulation of the spectral wave equation.

In Section 3, the spectral linear drag and stress formation theory is accomplished. The Miller-Pearce equations are linearised, and then transformed into Fourier normal modes. The spectral equations will provide the spectral presentations for the surface drag and wave stress in a straightforward manner and enable the expression of their spectral amplitudes in terms of the wave equation solution. In addition, the spectral wave equation of RZ07, initially formulated in log-pressure coordinates, is reintroduced in terms of the pressure vertical coordinate as this equation is later needed for the discussion of the Eliassen-Palm theorem. In Section 3.5 a major effort is made to check and refine the stress conservation conditions for NWM. To accomplish the NWM development, in Section 3.6 a discrete numerical realisation of the continuous up to this point buoyancy wave model is concisely discussed, including the 3D-discrete spectral wave equation presentation together with the appropriate top and surface boundary condition formulation.

The NWM is applied in Section 4 in the stratified atmosphere represented by a cold front which case is chosen in particular due to an enhanced wave generation ability by strong frontal winds. The main stress and drag characteristic features, including the conservation resp. variation with altitude, are studied numerically. Several novel orographic buoyancy wave properties, such as resonance rise in the wave, stress and drag spectra, and the wave-originated stratospheric quasi-turbulence (SQT) formation are established also here. 


\section{Drag formation in anelastic pressure-coordinate dynamics}

In this section, we introduce the non-hydrostatic, anelastic, pressure coordinate model of atmospheric dynamics to facilitate the pressure-coordinate presentation of wave drag basics.

\subsection{Modelling domain}

In the following, $S(V)$ represents a two- (three-) dimensional body of arbitrary configuration with area $\mathcal{S}$ (volume $\mathcal{V}$ ). The independent variables in pressure-coordinate dynamics are the horizontal coordinates $\mathbf{x}=\{x, y\}$, pressure $p$ (the vertical coordinate), and time $t$. The modelling domain $V$ is a cuboid with variable bottom height:

$$
V=S(0) \otimes\left\{0<p<p_{S}(\mathbf{x})=\overline{p_{s}}(1+\mu(\mathbf{x}))\right\},
$$

where

$$
S(0)=\left\{-L_{x}<x<L_{x}\right\} \otimes\left\{-L_{y}<y<L_{y}\right\}
$$

represents a rectangular region over the plane with total area $\mathcal{S}(0)=4 L_{x} L_{y}$ (see Fig. 1). In the equation, $p_{s}(\mathbf{x})$ is the orographic mean surface pressure. Strictly speaking, instead of the mean surface pressure, the actual total surface pressure $p_{s}(\mathbf{x})+p_{s}^{\prime}(\mathbf{x}, t)$, where $p_{s}^{\prime}(\mathbf{x}, t)$ is the variable part of the surface pressure, should be used in (2.1a). However, because of the smallness of $p_{s}^{\prime}(\mathbf{x}, t)$, the domain can be bounded by the fixed bottom surface $p_{s}$, considering the dynamically variable component $p_{s}^{\prime}$ only in the equations of motion (it is hidden in the geopotential $\varphi$; see below). In agreement with Fig. 1, area $S(p)$, occupied by the atmosphere at pressure level $p$, becomes smaller for levels that intersect with the

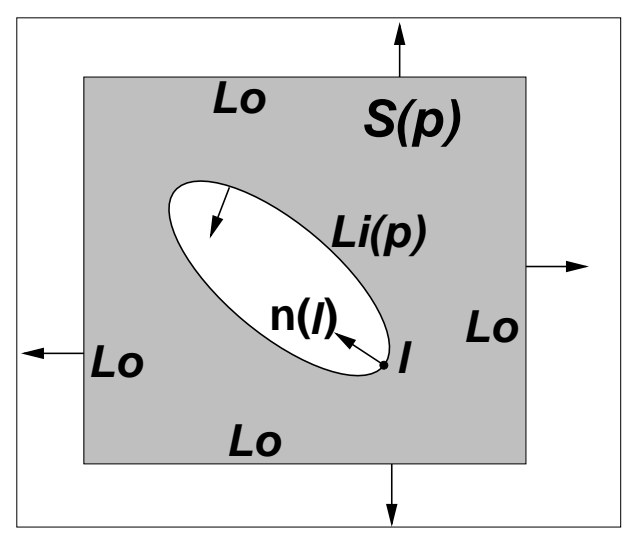

Figure 1: The grey area represents the 2D domain $S(p)$ occupied by the atmosphere on level $p$. The white area is the mountain-occupied domain on level $p, \operatorname{Li}(p)$ is the isobaric orography contour on level $p$, and $L o$ represents the lateral boundary contour of domain $V$. Arrows denote horizontal unit vectors, which are normal to the contours and directed out of the area $S(p)$. 
orography, forming isolines of baric orography:

$$
L(p)=\left\{p_{s}(\mathbf{x})=p\right\},
$$

whereas $\mathcal{S}(p)$ is a monotonic function of the pressure, limited as follows:

$$
0 \leq \mathcal{S}(p)=\int_{S(p)} d x d y \leq \mathcal{S}(0)=4 L_{x} L_{y} .
$$

As introduced in (2.1a), the area-mean surface pressure is

$$
\overline{p_{s}}=\frac{1}{\mathcal{S}(0)} \int_{S(0)} p_{S}(\mathbf{x}) d x d y,
$$

whereas the relative orographic surface pressure fluctuation is presented as

$$
\mu=\frac{p_{s}-\overline{p_{s}}}{\overline{p_{s}}} .
$$

\subsection{Equations of motion}

All fields are considered to be functions of the independent variables $\{\mathbf{x}, p, t\}$. The model uses temperature $T(\mathbf{x}, p, t)$, full geopotential (i.e. including both hydrostatic and nonhydrostatic components) $\varphi(\mathbf{x}, p, t)$, horizontal velocity $\mathbf{v}(\mathbf{x}, p, t)$, and vertical omega velocity $\omega(\mathbf{x}, p, t)$, as basic dynamic fields. Velocities $\mathbf{v}$ and $\omega$ are defined as

$$
\mathbf{v}=\frac{\mathrm{d} \mathbf{x}}{\mathrm{d} t}, \quad \omega=\frac{\mathrm{d} p}{\mathrm{~d} t}
$$

where $d / d t$ represents the material (substantial, Lagrangian) derivative:

$$
\frac{\mathrm{d}}{\mathrm{d} t}=\frac{\partial}{\partial t}+\mathbf{v} \cdot \nabla+\omega \frac{\partial}{\partial p}
$$

The components of the horizontal wind can be conveniently represented using the stream function $\Psi$ and flow potential $\Phi$ :

$$
\mathbf{v}=\nabla \Phi+\mathbf{e}_{z} \times \nabla \Psi,
$$

where $\mathbf{e}_{z}$ is the unit vertical vector. The divergence $D$ and vorticity (curl) $\xi$ that will be used hereafter are, respectively,

$$
D=\nabla \cdot \mathbf{v}=\frac{\partial v_{x}}{\partial x}+\frac{\partial v_{y}}{\partial y}, \quad \xi=\operatorname{curl}(\mathbf{v}) \equiv \frac{\partial v_{y}}{\partial x}-\frac{\partial v_{x}}{\partial y} .
$$

These terms can then be evaluated via $\Psi$ and $\Phi$ as

$$
D=\nabla^{2} \Phi, \quad \xi=\nabla^{2} \Psi .
$$


The basic equations in these notations are [42,45]:

$$
\begin{aligned}
& \frac{\mathrm{d} \omega}{\mathrm{d} t}=-\frac{p^{2}}{H^{2}}\left(\frac{\partial \varphi}{\partial p}+g \frac{H}{p}\right)+\frac{c_{v}}{c_{p}} \frac{\omega^{2}}{p}, \\
& \frac{\mathrm{d} \mathbf{v}}{\mathrm{d} t}=-\nabla W-f \mathbf{e}_{z} \times \nabla \Phi, \\
& \frac{\mathrm{d} T}{\mathrm{~d} t}=\frac{R}{c_{p}} \frac{T \omega}{p}, \\
& D+\frac{\partial \omega}{\partial p}=0,
\end{aligned}
$$

where $H=R T / g$ is the scale height, $g$ is the gravitational acceleration, $R, c_{v}, c_{p}$ are the gas constants, and $f$ is the locally constant ${ }^{\dagger}$ Coriolis parameter. The potential $W$ is defined as

$$
W=\varphi-f \Psi,
$$

$\varphi$ is the geopotential. The connection with the common presentation of the right-side forcing in (2.3b) is established by the relationship

$$
\nabla W+f \mathbf{e}_{z} \times \nabla \Phi=\nabla \varphi+f \mathbf{e}_{z} \times \mathbf{v} .
$$

For drag and momentum flux investigation, (2.3b) and (2.3d) are required (although (2.3a) through (2.3d) are involved in the derivation of the wave equation).

\subsection{Area- and volume-mean momenta}

The area-mean momentum on the pressure level $p$ is

$$
\mathbf{u}(p, t)=\overline{\mathbf{v}}(p, t)=\frac{1}{\mathcal{S}(p)} \int_{S(p)} \mathbf{v}(\mathbf{x}, p, t) d x d y .
$$

The volume-mean momentum $\mathbf{U}$ is

$$
\mathbf{U}(t)=\frac{1}{\mathcal{V}} \int_{S(0)} \int_{0}^{p_{s}(\mathbf{x})} \mathbf{v}(\mathbf{x}, p, t) d p d x d y,
$$

with the total domain volume

$$
\mathcal{V}=\int_{S(0)} \int_{0}^{p_{s}(\mathbf{x})} d p d x d y=\int_{S(0)} p_{S}(\mathbf{x}) d x d y=\int_{0}^{p_{s l}} \mathcal{S}(p) d p .
$$

The upper boundary of integration, $p_{s l}$, represents the sea-level standard pressure. The area $\mathcal{S}$ is considered to be zero for all points below the lowest orographic point $p_{\max } \leq p_{s l}$ and equal to the total area above the highest point $p_{m}$ on the orography:

$$
\left.\mathcal{S}\right|_{\left(p>p_{\max }\right)}=0,\left.\quad \mathcal{S}\right|_{\left(p<p_{m}\right)}=\mathcal{S}(0)=4 L_{x} L_{y} .
$$

\footnotetext{
${ }^{+}$Although $f$ depends on the coordinate $y$, it is considered a slow function; the beta effect is omitted, and $\nabla(f \psi)=f \nabla \psi$ is assumed where appropriate.
} 
Note the relationship

$$
\mathbf{U}=\langle\mathbf{u}\rangle \equiv \frac{1}{\mathcal{V}} \int_{0}^{p_{s l}} \mathcal{S}(p) \mathbf{u}(p) d p,
$$

which can be obtained from $(2.4 \mathrm{~b})$ by changing the order of integration and simultaneously defining the vertical mean of any $\psi(p)$ :

$$
\langle\psi\rangle=\frac{1}{\mathcal{V}} \int_{0}^{p_{s l}} \mathcal{S}(p) \psi(p) d p
$$

Note that the area mean $\mathbf{u}(2.4 \mathrm{a})$ as well as the area mean temperature

$$
\bar{T}(p, t)=\frac{1}{\mathcal{S}(p)} \int_{S(p)} T(\mathbf{x}, p, t) d x d y
$$

are employed in the NWM as sounding fields. In the following, all fields of interest are considered as sums of area means and fluctuation parts:

$$
\mathbf{v}=\mathbf{u}+\mathbf{v}^{\prime}, \quad T=\bar{T}+T^{\prime}, \quad W=\bar{W}+W^{\prime}, \quad \Psi=\bar{\Psi}+\Psi^{\prime}, \quad \Phi=\bar{\Phi}+\Phi^{\prime}, \quad \omega=\omega^{\prime} .
$$

In coarse numerical models, where the entire area $S(0)$ is treated as a single grid cell, the area mean functions represent the mean cell values, which vary gradually from cell to cell. In the NWM, the area-mean steady components are omitted where appropriate, which permits us to write

$$
\mathbf{u}(p)=\overline{\mathbf{v}}, \quad \bar{T}=\bar{T}(p), \quad \bar{W}=0, \quad \bar{\Psi}=0, \quad \bar{\Phi}=0 .
$$

\subsection{Average forcing on level $p$}

Although in the present paper we will deal with a linearised NWM, when introducing drag, it is advantageous to start with the full, non-linearised momentum balance equation (2.3b) in Eulerian flux form,

$$
\frac{\partial \mathbf{v}}{\partial t}=-\nabla W-f \mathbf{e}_{z} \times \nabla \Phi-\nabla \cdot(\mathbf{v v})-\frac{\partial \omega \mathbf{v}}{\partial p},
$$

because a premature linearisation would cause a substantial loss of detail. Averaging this equation over area $S(p)$ yields the balance equation for mean $p$-level momentum (2.4a):

$$
\frac{\partial \mathbf{u}(p, t)}{\partial t}=-\mathbf{a}(p, t)-\mathbf{b}(p, t)-\mathbf{c}(p, t),
$$

where the dynamic and advection components of the area-mean forcing are

$$
\begin{aligned}
& \mathbf{a}(p, t)=\frac{1}{\mathcal{S}(p)} \int_{S(p)} \nabla W d x d y=\overline{\nabla W^{\prime}}, \\
& \mathbf{b}(p, t)=\frac{1}{\mathcal{S}(p)} \int_{S(p)} f \mathbf{e}_{z} \times \nabla \Phi d x d y=f \mathbf{e}_{z} \times \overline{\nabla \Phi^{\prime}}, \\
& \mathbf{c}(p, t)=\frac{1}{\mathcal{S}(p)} \int_{S(p)}\left[\frac{\partial \omega \mathbf{v}}{\partial p}+\nabla \cdot(\mathbf{v v})\right] d x d y .
\end{aligned}
$$


By associating any area-mean forcing with a particular drag, the above-defined averaged forces will consequently be identified as the longitudinal orographic drag, the transverse orographic drag, and the wave drag which in following will be split also to the longitudinal and transverse constituents. The name 'orographic' is introduced for $\mathbf{a}$ and $\mathbf{b}$ because they arise on the inter-mountain pressure levels (i.e. on the levels $p>p_{m}$, where $p_{m}$ is the mean surface pressure at the highest point of orography) and vanish on the trans-mountain pressure-levels $\left(p<p_{m}\right)$, as will be shown below. The names 'longitudinal' and 'transverse' are introduced because the spectral amplitudes of consequent drag components become opposite and orthogonal to the horizontal wave vector in spectral space as also will be demonstrated later. Finally, name 'wave drag' for c will be justified in the course of the following transformation. Note that the presentation (2.5) is applied in the pressure and Coriolis drag definitions (2.6b) and (2.6c), but not in the wave drag definition (2.6d).

For further evaluation of area integrals in (2.6b) through (2.6d) and for coupling of drag forces with buoyancy waves, the motion is considered as satisfying the wave-type lateral boundary condition (WTLBC)

$$
\oint_{L o} \psi^{\prime} d l=0
$$

for each wave-originated field $\psi^{\prime}$ (representing all disturbed components denoted with a prime in (2.5a)). The contour integral over the outer contour Lo, which appears at the transformation of an area integral to a contour integral, is considered to be vanishing with the reasoning that either non-stationary $\psi$ is $2 \mathrm{D}$-periodic on the opposite outer boundaries of the domain in Fig. 1, or in the stationary case, it is created by obstacles inside the domain and disappears on the remote outer boundary Lo. In the case of low-resolution numerical dynamics, application of WTLBC is the only correct means for separating the numerically unresolved sub-grid forcing (like any drag), from the large-scale, numerically resolved forces. Indeed, in a low-resolution numerical model, the area $S(0)$ is considered as a single grid cell. Any field $\bar{\psi}+\psi^{\prime}$ consisting of the 'resolved', slow-changing component $\bar{\psi}$, and the fine-scale disturbance of local origin $\psi^{\prime}$ in (2.5a) is represented by the slow component only, whereas the fine-scale part remains unresolved and is disregarded. As an example, the averaged forcing on the right side of (2.6b) splits into

$$
\mathbf{a}=\overline{\nabla W^{\prime}}+\overline{\nabla \bar{W}}
$$

where the representation (2.6b) holds for the first term, whereas the second satisfies the representation

$$
\overline{\nabla \bar{W}}=\frac{1}{\mathcal{S}(0)} \oint_{L o} \bar{W} \mathbf{n} d l
$$

which, incidentally, defines the volume-element approximation of the gradient $\nabla \bar{W}$ in grid-cell $S(0)$. Application of WTLBC eliminates such large-scale forcing and provides sub-grid drag treatment in the pure mode. 
In the case of (2.6b) and (2.6c), integration over the area converts, with the help of the Gauss formula, to the line integrals over the bounding contours, which then simplify to line integrals over the internal contour $L_{i}(p)$ (internal contour $L i$ can consist of a single loop like in Fig. 1 or of several (multiple) loops in the case of a more complex orography), if $W^{\prime}$ and $\Phi^{\prime}$ satisfy WTLBC:

$$
\begin{aligned}
& \int_{S(p)} \nabla W^{\prime} d x d y=\oint_{L i(p)+L o} W_{S}^{\prime} \mathbf{n} d l=\oint_{L i(p)} W_{S}^{\prime} \mathbf{n} d l, \\
& \int_{S(p)} f \mathbf{e}_{z} \times \nabla \Phi^{\prime} d x d y=\oint_{L o+L i(p)} f \Phi^{\prime} \mathbf{e}_{z} \times \mathbf{n} d l=f \mathbf{e}_{z} \times \oint_{L i(p)} \mathbf{n}(l) \Phi_{S}^{\prime} d l .
\end{aligned}
$$

Here $\mathbf{n}(l)$ is the outer normal of the contour at any particular contour point $l$ (see Fig. 1). Thus, (2.6b) and (2.6c) transform to the line integral over internal contour $L i$ :

$$
\begin{aligned}
& \mathbf{a}=\frac{1}{\mathcal{S}(p)} \oint_{L i(p)} W_{S}^{\prime} \mathbf{n} d l, \\
& \mathbf{b}=\frac{f}{\mathcal{S}(p)} \mathbf{e}_{z} \times \oint_{L i(p)} \mathbf{n} \Phi_{S}^{\prime} d l .
\end{aligned}
$$

As seen, both orographic drag components $\mathbf{a}$ and $\mathbf{b}$ act on the averaged (area-mean) horizontal momentum on the inter-mountain levels with the finite $L_{i}(p)$ and vanish on the trans-mountain levels where $L_{i}(p)$ disappears (becomes zero by length). They can vanish also on the inter-mountain levels, if the potential fluctuations $W_{s}^{\prime}$ and $\Phi_{s}^{\prime}$ become zero along particular contour. In NWM all fields, including $W_{s}^{\prime}$ and $\Phi_{s}^{\prime}$, are expressed via the wave equation solution (which is $\omega$ ) and become disturbed in concert. Thus, the wave generation always supports also the orographic drag creation, which however vanishes on the trans-mountain levels.

As $W_{s}^{\prime}$ is proportional to the dynamic surface pressure fluctuation in the same contour point, a corresponds to the mean pressure force on level $p$. For simplest $2 \mathrm{D}$ flow over a perpendicular 2D mountain, $\mathbf{a}$ is proportional to the dynamic pressure difference between front and lee side of the mountain on level $p$ and is directed downstream, so that $-\mathbf{a}$ in (2.6a) will slow down the mean momentum $\mathbf{u}(p, t)$. That is, the longitudinal orographic drag tries to stagnate the inter-mountain flow and create a low-level blocking. In similar conditions, the transverse drag $\mathbf{b}$ is perpendicular to the main flow and will accelerate the mean circulation in parallel with the mountain crest.

The wave drag in (2.6d) can be converted to

$$
\mathbf{c}=\frac{1}{\mathcal{S}(p)} \frac{\partial \mathcal{S}(p) \gamma(p, t)}{\partial p}
$$

where the wave stress vector

$$
\gamma(p, t)=\frac{1}{\mathcal{S}(p)} \int_{S(p)} \omega \mathbf{v} d x d y=\overline{\omega \mathbf{v}}=\overline{\omega \mathbf{v}^{\prime}}
$$


represents the area-mean momentum flux on level $p$. To prove the presentation in (2.7a) and $(2.7 \mathrm{~b})$, integration by parts and the Gaussian formula must be appropriately applied in $(2.6 \mathrm{~d})$ :

$$
\begin{aligned}
& \int_{S(p)}\left[\frac{\partial \omega \mathbf{v}}{\partial p}+\nabla \cdot(\mathbf{v v})\right] d x d y \\
= & \frac{\partial}{\partial p} \int_{S(p)} \omega \mathbf{v} d x d y+\oint_{L o} \mathbf{v}(\mathbf{v} \cdot \mathbf{n}(\mathbf{1})) d l+\oint_{L i(p)} \mathbf{v}_{\mathbf{s}}\left[\mathbf{v}_{\mathbf{s}} \cdot \mathbf{n}+\frac{\omega_{S}}{\left|\nabla p_{S}\right|}\right] d l .
\end{aligned}
$$

Here, it is considered that the line integral over the outer contour Lo vanishes if $\mathbf{v}^{\prime}$ satisfies the WTLBC. The second line integral over $\operatorname{Li}(p)$ annihilates due to the free-slip boundary condition on the surface

$$
\omega_{s}=\mathbf{v}_{s} \cdot \nabla p_{s}, \quad \text { yielding } \quad \frac{\omega_{s}}{\left|\nabla p_{s}\right|}=\frac{\mathbf{v}_{s} \cdot \nabla p_{s}}{\left|\nabla p_{s}\right|}=-\mathbf{v}_{s} \cdot \mathbf{n} .
$$

Note that the free-slip boundary condition ignores any local tendency of the pressure, hence $p_{s}(\mathbf{x})$ represents the time-independent mean part of the surface pressure, while the tendency of the variable part $\partial p_{s}^{\prime} / \partial t$ is considered negligible.

The wave stress $(2.7 \mathrm{~b})$ can be further decomposed with the help of (2.2c) to the longitudinal and transverse stresses:

$$
\gamma=\tau+\sigma, \quad \tau=\overline{\omega \nabla \Phi^{\prime}}, \quad \sigma=\mathbf{e}_{z} \times \overline{\omega \nabla \Psi^{\prime}}
$$

This enables analogous decomposition of the wave drag:

$$
\mathbf{c}=\mathbf{c}_{l}+\mathbf{c}_{t}, \quad \mathbf{c}_{l}=\frac{1}{\mathcal{S}} \frac{\partial \mathcal{S} \tau}{\partial p}, \quad \mathbf{c}_{t}=\frac{1}{\mathcal{S}} \frac{\partial \mathcal{S} \sigma}{\partial p} .
$$

The names 'longitudinal' and 'transverse' stress are justified here also because the spectral amplitudes of consequent stress and drag components become opposite and orthogonal to the horizontal wave vector in spectral space (as will be demonstrated later).

As discussed above, $\mathbf{a}$ and $\mathbf{b}$ are altitude-dependent according to (2.6)' and become identically zero for trans-mountain pressure levels:

$$
\mathbf{a}(p, t) \equiv 0, \quad \mathbf{b}(p, t) \equiv 0, \quad p<p_{m} .
$$

Thus, the balance equation (2.6a) simplifies for trans-mountain levels, where $\mathcal{S}(p)=\mathcal{S}(0)$, to

$$
\frac{\partial \mathbf{u}(p, t)}{\partial t}=-\frac{\partial \tau(p, t)}{\partial p}-\frac{\partial \sigma(p, t)}{\partial p}, \quad p<p_{m} .
$$




\subsection{Surface drag}

Multiplication of (2.6a) with $\mathcal{S}(p)$, integration over $p$, and division by $\mathcal{V}$ yields the balance equation for volume-mean momentum:

$$
\frac{\partial \mathbf{U}}{\partial t}=-\mathbf{A}-\mathbf{B}-\mathbf{C}
$$

The first and second terms can be presented with the help of (2.6b) and (2.6c):

$$
\begin{aligned}
& \mathbf{A}=\langle\mathbf{a}\rangle=\frac{1}{\mathcal{V}} \int_{S(0)} \int_{0}^{p_{S}(\mathbf{x})} \nabla W^{\prime} d p d x d y \\
& \mathbf{B}=\langle\mathbf{b}\rangle=\frac{f}{\mathcal{V}} \int_{S(0)} \int_{0}^{p_{S}(\mathbf{x})} \mathbf{e}_{z} \times \nabla \Phi^{\prime} d p d x d y .
\end{aligned}
$$

They can be presented with the help of the identity

$$
\int_{0}^{p_{s}} \nabla \psi d p=-\psi_{s} \nabla p_{s}+\nabla \int_{0}^{p_{s}} \psi d p=-\psi_{s} \bar{p}_{s} \nabla \mu+\nabla \int_{0}^{p_{s}} \psi d p,
$$

with the additional assumption that $W^{\prime}$ and $\Phi^{\prime}$ satisfy WTLBC, as

$$
\mathbf{A}=-\overline{\left(W_{s}^{\prime} \nabla \mu\right)}, \quad \mathbf{B}=-f \overline{\left(\Phi_{s}^{\prime} \mathbf{e}_{z} \times \nabla \mu\right)} .
$$

The first formula defines the longitudinal surface drag, coinciding with the earlier surface drag definition $[12,46]$ if $W_{s}$ can be expressed in terms of the wave-related surface pressure fluctuation. Thus, the vertically averaged longitudinal orographic drag yields the longitudinal surface drag. By analogy $\mathbf{B}$ can be named the transverse surface drag. In some studies it is called also 'orographic lift' [47].

The third term in (2.9a) vanishes:

$$
\mathrm{C}=0 \text {, }
$$

because

$$
\mathcal{V} \mathbf{C}=\int_{0}^{p_{s l}} \mathcal{S}(p) \mathbf{c} d p=\int_{0}^{p_{s l}} \frac{\partial \mathcal{S}(p) \gamma(p, t)}{\partial p} d p=\int_{S\left(p_{s l}\right)} \omega \mathbf{v} d x d y-\int_{S(0)} \omega \mathbf{v} d x d y=0,
$$

as $\mathcal{S}\left(p_{s l}\right)=0$ in the first area integral and $\left.\omega\right|_{p=0}=0$ in the second. The nullification of the vertically averaged wave drag is an important property of the inviscid atmosphere, indicating that the vertical momentum flux cannot change the overall horizontal momentum of the atmosphere but merely redistributes it among different levels.

Though A and B are evaluated according to (2.9d) as area-mean integrals over underlying surface, and thus seemingly should affect the near-surface momentum, actually they are defined in (2.9b)-(2.9c) as the volume means. Thus, at the drag parameterisation they have to be applied in any particular grid-cell at all pressure levels alike, supplementing the momentum equation (2.3b) with the constant forcing $-\mathbf{A}-\mathbf{B}$ on the right 
side. Such coarse surface drag parametrisation is justified only if the vertical resolution of the numerical model is very coarse (an one-layer shallow-water model, as an example). Meanwhile, $\mathbf{A}$ and $\mathbf{B}$ can be equally evaluated as $\mathbf{A}=\langle\mathbf{a}\rangle, \quad \mathbf{B}=\langle\mathbf{b}\rangle$, where vertical averaging $\langle\psi\rangle$ is defined by (2.4e), whilst the orographic drag vectors $\mathbf{a}$ and $\mathbf{b}$ are computed from $(2.6 b)^{\prime}$ and (2.6c)'. As it was established before, $\mathbf{a}$ and $\mathbf{b}$ are highly altitude-dependent, affecting the inter-mountain mean momentum and vanishing on the trans-mountain levels $p<p_{m}$. That gives an idea to modify the surface drag concept, confining the vertical averaging to the inter-mountain levels

$$
\begin{aligned}
& \mathbf{A}\left(p_{m}\right)=\frac{1}{\mathcal{V}\left(p_{m}\right)} \int_{p_{m}}^{p_{s l}} \mathcal{S}(p) \mathbf{a}(p) d p, \\
& \mathbf{B}\left(p_{m}\right)=\frac{1}{\mathcal{V}\left(p_{m}\right)} \int_{p_{m}}^{p_{s l}} \mathcal{S}(p) \mathbf{b}(p) d p, \\
& \mathcal{V}\left(p_{m}\right)=\int_{p_{m}}^{p_{s l}} \mathcal{S}(p) d p,
\end{aligned}
$$

and apply the surface drag to the inter-mountain air layer only. We are not aware of any analogous modification of the surface drag before. In this paper we will still confine the treatment with the common definitions (2.9d), because the modified surface drags can be find via those using

$$
\mathbf{A}\left(p_{m}\right)=\frac{\mathcal{V}}{\mathcal{V}\left(p_{m}\right)} \mathbf{A}, \quad \mathbf{B}\left(p_{m}\right)=\frac{\mathcal{V}}{\mathcal{V}\left(p_{m}\right)} \mathbf{B}
$$

\section{Spectral numerical wave model}

Of prime interest for wave-related drag study are the longitudinal and transverse surface drag (2.9d), the longitudinal and transverse wave stress (2.7d), and the longitudinal and transverse wave drag as defined in $(2.7 \mathrm{e}),(2.8 \mathrm{~b})$. To take their wave origin into consideration explicitly, it becomes vital to express their underlying fields $W^{\prime}, \Phi^{\prime}$, and $\Psi^{\prime}$ in the framework of the linear NWM. For this purpose, a concise revisiting of the linear wave model as initiated in RZ07 is required.

\subsection{Linear equations}

Using (2.5) and separating the linear and nonlinear parts of the substantial derivative

$$
\begin{aligned}
& \frac{\mathrm{d}}{\mathrm{d} t}=\frac{\mathrm{d}_{0}}{\mathrm{~d} t}+\frac{\mathrm{d}^{\prime}}{\mathrm{d} t^{\prime}} \\
& \frac{\mathrm{d}_{0}}{\mathrm{~d} t}=\frac{\partial}{\partial t}+\mathbf{u}(p) \cdot \nabla, \quad \frac{\mathrm{d}^{\prime}}{\mathrm{d} t}=\mathbf{v}^{\prime} \cdot \nabla+\omega \frac{\partial}{\partial p^{\prime}}
\end{aligned}
$$


the kinematic relationships (2.2c) through (2.2e) reduce to

$$
\begin{aligned}
& \mathbf{v}^{\prime}=\nabla \Phi^{\prime}+\mathbf{e}_{z} \times \nabla \Psi^{\prime}, \\
& \xi=\operatorname{curl}\left(\mathbf{v}^{\prime}\right) \equiv \frac{\partial v_{y}^{\prime}}{\partial x}-\frac{\partial v_{x}^{\prime}}{\partial y}, \\
& \nabla^{2} \Phi^{\prime}=\nabla \cdot \mathbf{v}^{\prime}=-\frac{\partial \omega}{\partial p}, \\
& \nabla^{2} \Psi^{\prime}=\xi
\end{aligned}
$$

whereas the system represented by (2.3) withdraws to the linear equations (which means that all second-order small constructs such as $\psi_{1}^{\prime} \psi_{2}^{\prime}$ and $d^{\prime} \psi^{\prime} / d t$ are omitted) for vertical velocity $\omega$, temperature fluctuation $T^{\prime}$, and vorticity $\xi$ :

$$
\begin{aligned}
& \frac{\mathrm{d}_{0} \omega}{\mathrm{d} t}=-\frac{p^{2}}{H^{2}}\left(\frac{\partial \varphi^{\prime}}{\partial p}+\frac{R T^{\prime}}{p}\right), \\
& \frac{\mathrm{d}_{0} \xi}{\mathrm{d} t}=f \frac{\partial \omega}{\partial p}+\mathbf{e}_{z}\left(\frac{\partial \mathbf{u}}{\partial p} \times \nabla \omega\right), \\
& \frac{\mathrm{d}_{0}}{\mathrm{~d} t} \frac{\partial \omega}{\partial p}=\nabla^{2} W^{\prime}+\frac{\partial \mathbf{u}}{\partial p} \cdot \nabla \omega, \\
& \frac{\mathrm{d}_{0} T^{\prime}}{\mathrm{d} t}=\frac{\theta \omega}{p} \\
& W^{\prime}=\varphi^{\prime}-f \Psi^{\prime},
\end{aligned}
$$

where $H=R \bar{T} / g, \quad \theta=\left(R / c_{p}\right) \bar{T}-p \partial \bar{T} / \partial p$. System (3.2) is the basis for the wave equation and drag component presentation via the wave equation solution.

\subsection{Spectral equations}

Though the drag and stress components are expressed in common Cartesian coordinates $x, y$, the NWM itself is essentially a spectral model. Thus, system (3.2) must be transformed into Fourier space with the following wave equation deduction and spectral representation of stress and drag. For this, all functions of interest are presented as normalmode series in horizontal coordinates and time:

$$
\psi(\mathbf{x}, p, t)=\sum_{v, \mathbf{k}} \widehat{\psi}_{\mathbf{k}}^{v}(p) \mathrm{e}^{\mathrm{i}[v t-(\mathbf{k} \cdot \mathbf{x})]}=\sum_{\mathbf{k}} \widehat{\psi}_{\mathbf{k}}^{0}(p) \mathrm{e}^{-\mathrm{i}(\mathbf{k} \cdot \mathbf{x})}+\sum_{v \neq 0, \mathbf{k}} \widehat{\psi}_{\mathbf{k}}^{v}(p) \mathrm{e}^{\mathrm{i}[v t-(\mathbf{k} \cdot \mathbf{x})]},
$$

with corresponding surface projections of

$$
\psi_{s}(\mathbf{x}, t) \equiv \psi\left(\mathbf{x}, p_{s}(\mathbf{x}), t\right)=\sum_{v, \mathbf{k}} \widehat{\psi}_{s \mathbf{k}}^{v} \mathrm{e}^{\mathrm{i}[v t-(\mathbf{k} \cdot \mathbf{x})]}
$$


$\widehat{\psi}_{\mathbf{k}}^{v}$ represents the spectral amplitudes or normal modes of $\psi$ with frequency (wave equation eigenvalue) $v$ and discrete wave vector $\mathbf{k}$ :

$$
\mathbf{k}=\left\{k_{x}, k_{y}\right\} ; \quad k_{x}=j_{x} \pi / L_{x}, \quad k_{y}=j_{y} \pi / L_{y} ; \quad j_{x}, j_{y}=0, \pm 1, \pm 2, \pm 3, \cdots .
$$

The summation in (3.3) proceeds over the discrete set of $\left\{k_{x}, k_{y}, v\right\}$. Case $v=0$ yields the stationary mode. The partial sum $\sum_{\mathbf{k}} \widehat{\psi}_{\mathbf{k}}^{0}(p) \mathrm{e}^{-\mathrm{i}(\mathbf{k} \cdot \mathbf{x})}$ in (3.3a) represents the stationary part of $\psi$. The relationships in (3.1) are transformed to relationships for normal modes:

$$
\begin{aligned}
& \widehat{\mathbf{v}}_{\mathbf{k}}^{v}=-\mathrm{i} \mathbf{k} \widehat{\Phi}_{\mathbf{k}}^{v}-\mathrm{i}\left(\mathbf{e}_{z} \times \mathbf{k}\right) \widehat{\Psi}_{\mathbf{k}}^{v} \\
& \widehat{\zeta}_{\mathbf{k}}^{v}=-\mathrm{i} k_{x}\left(\widehat{v}_{y}^{\prime}\right)_{\mathbf{k}}^{v}+\mathrm{i} k_{y}\left(\widehat{v}_{x}^{\prime}\right)_{\mathbf{k}^{\prime}}^{v} \\
& k^{2} \widehat{\Phi}_{\mathbf{k}}^{v}=\frac{\partial \widehat{\omega}_{\mathbf{k}}^{v}}{\partial p} \\
& k^{2} \widehat{\Psi}_{\mathbf{k}}^{v}=-\widehat{\zeta}_{\mathbf{k}^{\prime}}^{v}
\end{aligned}
$$

whereas the equations in (3.2) transform to

$$
\begin{aligned}
& \mathrm{i} n_{\mathbf{k}}^{v} \widehat{\omega}_{\mathbf{k}}^{v}=-\frac{p^{2}}{H^{2}}\left(\frac{\partial \widehat{\varphi}_{\mathbf{k}}^{v}}{\partial p}+\frac{R \widehat{T}_{\mathbf{k}}^{v}}{p}\right), \\
& \mathrm{i} n_{\mathbf{k}}^{v} \widehat{\xi}_{\mathbf{k}}^{v}=f \frac{\partial \widehat{\omega}_{\mathbf{k}}^{v}}{\partial p}+\mathrm{i} v_{\mathbf{k}}^{2}(p) \frac{\widehat{\omega}_{\mathbf{k}}^{v}}{p} \\
& \mathrm{i} n_{\mathbf{k}}^{v} \frac{\partial \widehat{\omega}_{\mathbf{k}}^{v}}{\partial p}=-k^{2} \widehat{W}_{\mathbf{k}}^{v}-\mathrm{i} v_{\mathbf{k}}^{1} \frac{\widehat{\omega}_{\mathbf{k}}^{v}}{p} \\
& \mathrm{i} n_{\mathbf{k}}^{v} T_{\mathbf{k}}^{\prime v}=\frac{\theta \widehat{\omega}_{\mathbf{k}}^{v}}{p} \\
& \widehat{W}_{\mathbf{k}}^{v}=\widehat{\varphi}_{\mathbf{k}}^{v}-f \widehat{\Psi}_{\mathbf{k}^{\prime}}^{v}
\end{aligned}
$$

where the intrinsic frequency

$$
n_{\mathbf{k}}^{v}=n_{\mathbf{k}}^{v}(p)=v-v_{\mathbf{k}}^{0}, \quad v_{\mathbf{k}}^{0}=\mathbf{k} \cdot \mathbf{u}(p)
$$

represents the Fourier transform of $-\mathrm{i} d_{0} / d t$. Other new parameters with frequency dimension are

$$
v_{\mathbf{k}}^{1}=p \frac{\partial \mathbf{k} \cdot \mathbf{u}}{\partial p}=p \frac{\partial v_{\mathbf{k}}^{0}}{\partial p}, \quad v_{\mathbf{k}}^{2}=p \frac{\partial\left(k_{x} u_{y}-k_{y} u_{x}\right)}{\partial p}=\mathbf{e}_{z} \cdot\left(\mathbf{k} \times p \frac{\partial \mathbf{u}}{\partial p}\right) .
$$

\subsection{Spectral wave equation}

If we use mutual eliminations in (3.5) (details see RZ07), a wave equation proceeds for $\widehat{\omega}_{\mathbf{k}}^{v}$ :

$$
p^{2} \frac{\partial^{2} \widehat{\omega}_{\mathbf{k}}^{v}}{\partial p^{2}}-Q_{\mathbf{k}}^{v} p \frac{\partial \widehat{\omega}_{\mathbf{k}}^{v}}{\partial p}+\Lambda_{\mathbf{k}}^{v} \widehat{\omega}_{\mathbf{k}}^{v}=0
$$


with coefficients

$$
\begin{aligned}
& Q_{\mathbf{k}}^{v}(p)=\frac{f^{2}}{\left(n_{\mathbf{k}}^{v}\right)^{2}-f^{2}}\left(\frac{v_{\mathbf{k}}^{1}}{n_{\mathbf{k}}^{v}}+\frac{\mathrm{i} v_{\mathbf{k}}^{2}}{f}\right), \\
& \Lambda_{\mathbf{k}}^{v}(p)=\Re \Lambda_{\mathbf{k}}^{v}+\mathrm{i} \Im \Lambda_{\mathbf{k}}^{v}, \\
& \Re \Lambda_{\mathbf{k}}^{v}=\frac{H^{2} k^{2}\left(N^{2}-\left(n_{\mathbf{k}}^{v}\right)^{2}\right)+n_{\mathbf{k}}^{v} v_{\mathbf{k}}^{4}}{\left(n_{\mathbf{k}}^{v}\right)^{2}-f^{2}}, \Im \Lambda_{\mathbf{k}}^{v}=\frac{\mathrm{i} f}{\left(n_{\mathbf{k}}^{v}\right)^{2}-f^{2}}\left(v_{\mathbf{k}}^{2}-\frac{v_{\mathbf{k}}^{1}}{n_{\mathbf{k}}^{v}} v_{\mathbf{k}}^{2}-v_{\mathbf{k}}^{3}\right), \\
& N(p)=\frac{\sqrt{R \theta}}{H}=\sqrt{\frac{g}{H}\left(\frac{R}{c_{p}}-\frac{p}{H} \frac{\partial H}{\partial p}\right)} \\
& v_{\mathbf{k}}^{3}=p \frac{\partial v_{\mathbf{k}}^{2}}{\partial p}, \quad v_{\mathbf{k}}^{4}=p^{2} \frac{\partial^{2} \mathbf{k} \cdot \mathbf{u}}{\partial p^{2}}=p \frac{\partial v_{\mathbf{k}}^{1}}{\partial p}-v_{\mathbf{k}}^{1} .
\end{aligned}
$$

Here and further $\Re Z$ and $\Im Z$ will denote the real and imaginary parts of any complex $Z$. Eq. (3.7a), which presents the pressure-coordinate generalisation of the Scorer equation [48] for arbitrary shear wind and thermal stratification. It is equivalent to the equation of RZ07, despite appearing slightly different here. The main difference is that the nonstationary option $v \neq 0$ is included, and the pressure vertical co-ordinate is used instead of the log-pressure height. The main parameter of the equation is $\Re \Lambda(p) ; \Re \Lambda / H^{2}$ is often called the Scorer parameter. If the wind changes slow with the altitude, so that $v_{\mathbf{k}}^{1}, v_{\mathbf{k}}^{2} \rightarrow 0$, then $Q$ and $\Im \Lambda$ can be considered small perturbations and omitted, in which case the wave equation simplifies to

whereas

$$
p^{2} \frac{\partial^{2} \widehat{\omega}_{\mathbf{k}}^{v}}{\partial p^{2}}+\Lambda_{\mathbf{k}}^{v} \widehat{\omega}_{\mathbf{k}}^{v}=0
$$

$$
\Lambda_{\mathbf{k}}^{v}(p)=H^{2} k^{2} \frac{N^{2}-\left(n_{\mathbf{k}}^{v}\right)^{2}}{\left(n_{\mathbf{k}}^{v}\right)^{2}-f^{2}}
$$

becomes real. The term proportional to $\partial^{2} \mathbf{u} / \partial p^{2}$ in the nominator of $\Re \Lambda$ in (3.7c) is omitted in (3.8b) for the same reason. If, in addition, the wind is constant, then $\Lambda_{\mathbf{k}}^{v}$ becomes constant also. However, the terms $Q$ and $\Im \Lambda$ cannot neglected for more rigorous wind profiles automatically. As we will see later, they become really substantial for shear wind, causing notable modifications of wave amplitudes and stress conservation properties. For stationary modes $v=0$, there do exist critical combinations of wave vectors $\mathbf{k}$ and wind vector $\mathbf{u}(p)$

$$
\left(n_{\mathbf{k}}^{0}\right)^{2}-f^{2}=0 \rightarrow \mathbf{k} \cdot \mathbf{u}= \pm f \rightarrow k=f /\left|\mathbf{n}_{\mathbf{k}} \cdot \mathbf{u}(p)\right|,
$$

for which $n_{\mathbf{k}}=\mathbf{k} / k$ is non-orthogonal to the wind $\mathbf{u}(p)$ (an exception is $f=0$, in which case either $\mathbf{k}=0$ or $\mathbf{k}$ and $\mathbf{u}(p)$ strictly transverse, indeed). Levels $p$ (if they do exist for given k) are called critical. On the critical levels both $\Lambda$ and $Q$ become infinite. For solutions $\widehat{\omega}$ with continuous first derivative $\partial \widehat{\omega} / \partial p$ and finite (though probably discontinuous) second derivative $\partial^{2} \widehat{\omega} / \partial p^{2}$, that will mean nullification of the solution on any critical level. 


\subsection{Spectral presentation of stress and drag}

The wave stress (2.7d), wave drag (2.7e), and surface drag (2.9d) components all represent area means of the kind

$$
\overline{\psi_{1} \psi_{2}}(p)=\frac{1}{4 L_{x} L_{y}} \int_{S(0)} \psi_{1}(\mathbf{x}, p) \psi_{2}(\mathbf{x}, p) d x d y .
$$

To consider also the non-stationary solutions of the wave equations in a physically relevant way, it is advantageous to include time averaging into the definition of the mean:

$$
\overline{\psi_{1} \psi_{2}}(p)=\frac{1}{8 L_{x} L_{y} T} \int_{-T}^{T} \int_{S(0)} \psi_{1}(\mathbf{x}, p, t) \psi_{1}(\mathbf{x}, p, t) d x d y d t,
$$

where $T$ is half the time averaging interval, which is sufficiently long to filter out shorttime oscillations but still short compared with the characteristic time-scale of the mean momentum $\mathbf{u}$ in the balance equation (3.6). For $\psi_{1}$ and $\psi_{2}$, presented as series (3.3), the formula (3.9a) becomes

$$
\overline{\psi_{1} \psi_{2}}(p)=\sum_{v, \mathbf{k}} \widehat{\psi}_{1 \mathbf{k}}^{v}(p)\left(\widehat{\psi}_{2 \mathbf{k}}^{v}(p)\right)^{*} .
$$

If at least one of the fields $\psi_{1}$ or $\psi_{2}$ is stationary (the second may also contain an oscillating part), (3.9a) and (3.9b) automatically revert to the former case of areal averaging:

$$
\overline{\psi_{1} \psi_{2}}(p)=\frac{1}{4 L_{x} L_{y}} \int_{S(0)} \psi_{1}(\mathbf{x}, p) \psi_{2}(\mathbf{x}, p) d x d y=\sum_{\mathbf{k}} \widehat{\psi}_{1 \mathbf{k}}^{0}(p) \widehat{\psi}_{2 \mathbf{k}}^{0 *}(p) .
$$

The spectral representations for surface drag (2.9d) and wave stress (2.7d) are

$$
\begin{aligned}
& \mathbf{A}=-\mathrm{i} \sum_{\mathbf{k}} \mathbf{k} \widehat{W}_{s \mathbf{k}}^{0} \widehat{\mu}_{\mathbf{k}}^{*}, \quad \mathbf{B}=-\mathrm{i} f \sum_{\mathbf{k}}\left(\mathbf{e}_{z} \times \mathbf{k}\right) \widehat{\Phi}_{s \mathbf{k}}^{0} \widehat{\mu}_{\mathbf{k}}^{*}, \\
& \boldsymbol{\tau}=\mathrm{i} \sum_{\nu, \mathbf{k}} \mathbf{k} \widehat{\omega}_{\mathbf{k}}^{v}(p)\left(\widehat{\Phi}_{\mathbf{k}}^{v}\right)^{*}, \quad \sigma=\mathrm{i} \sum_{\nu, \mathbf{k}}\left(\mathbf{e}_{z} \times \mathbf{k}\right) \widehat{\omega}_{\mathbf{k}}^{v}(p)\left(\widehat{\Psi}_{\mathbf{k}}^{v}\right)^{*},
\end{aligned}
$$

with coefficients from (3.4) and (3.5):

$$
\widehat{W}_{\mathbf{k}}^{v}=\frac{-\mathrm{i}}{k^{2}}\left(n_{\mathbf{k}}^{v} \frac{\partial \widehat{\omega}_{\mathbf{k}}^{v}}{\partial p}+v_{\mathbf{k}}^{1} \frac{\widehat{\omega}_{\mathbf{k}}^{v}}{p}\right), \quad \widehat{\Phi}_{\mathbf{k}}^{v}=\frac{1}{k^{2}} \frac{\partial \widehat{\omega}_{\mathbf{k}}^{v}}{\partial p}, \quad \widehat{\Psi}_{\mathbf{k}}^{v}=\frac{1}{k^{2} n_{\mathbf{k}}^{v}}\left(\mathrm{i} f \frac{\partial \widehat{\omega}_{\mathbf{k}}^{v}}{\partial p}-\frac{v_{\mathbf{k}}^{2} \widehat{\omega}_{\mathbf{k}}^{v}}{p}\right) .
$$

For separation of orography and stratification effects in the final spectral formulations, it is advantageous to re-norm the spectral solution of the wave equation as follows:

$$
\widehat{\omega}_{\mathbf{k}}^{v}(p)=-\mathbf{i}\left(\mathbf{u}_{s} \cdot \mathbf{k}\right)\left\{\begin{array}{ll}
\widehat{\mu}_{\mathbf{k}}, & v=0 \\
\alpha_{\mathbf{k}^{\prime}}^{v} & v \neq 0
\end{array}\right\} \Omega_{\mathbf{k}}^{v}(p), \quad \Omega_{\mathbf{k}}^{v}\left(\bar{p}_{s}\right)=\Omega_{s \mathbf{k}}^{v}= \begin{cases}\bar{p}_{s^{\prime}} & v=0, \\
0, & v \neq 0,\end{cases}
$$

where $\widehat{\mu}_{\mathbf{k}}$ represents the spectrum of relative height of orography $\mu(\mathbf{x})$, whereas $\alpha_{\mathbf{k}}^{v}$ are the amplitudes of the non-stationary solution, which are specified from the initial conditions 
for $\omega$. Because the stationary solution satisfies the free slip condition on the surface (2.7c), which in normal modes becomes

$$
\widehat{\omega}_{s \mathbf{k}}^{0}=-\mathrm{i}\left(\mathbf{u}_{s} \cdot \mathbf{k}\right) \bar{p}_{s} \widehat{\mu}_{\mathbf{k}},
$$

whereas for non-stationary modes

$$
\widehat{\omega}_{s \mathbf{k}}^{v}=0,
$$

the new normal modes $\Omega_{\mathbf{k}}^{v}(p)$ are expected to satisfy the lower boundary condition in (3.11a).

Using (3.10c) and (3.11), the (3.5) in series (3.10) can be presented

$$
\begin{aligned}
& \mathbf{A}=\sum_{\mathbf{k}} \mathbf{A}_{\mathbf{k}}\left|\widehat{\mu}_{\mathbf{k}}\right|^{2}, \quad \mathbf{B}=\sum_{\mathbf{k}} \mathbf{B}_{\mathbf{k}}\left|\widehat{\mu}_{\mathbf{k}}\right|^{2}, \\
& \boldsymbol{\tau}(p)=\sum_{\mathbf{k}} \boldsymbol{\tau}_{\mathbf{k}}^{0}(p)\left|\mu_{\mathbf{k}}\right|^{2}+\sum_{\nu \neq 0, \mathbf{k}} \tau_{\mathbf{k}}^{v}(p)\left|\alpha_{\mathbf{k}}^{v}\right|^{2}, \\
& \sigma(p)=\sum_{\mathbf{k}} \sigma_{\mathbf{k}}^{0}(p)\left|\mu_{\mathbf{k}}\right|^{2}+\sum_{\nu \neq 0, \mathbf{k}} \sigma_{\mathbf{k}}^{v}(p)\left|\alpha_{\mathbf{k}}^{v}\right|^{2},
\end{aligned}
$$

with the spectral amplitudes as

$$
\begin{aligned}
& \mathbf{A}_{\mathbf{k}}=\mathbf{k} \frac{\left(\mathbf{u}_{\mathbf{s}} \cdot \mathbf{k}\right)^{2}}{k^{2}} \Im\left(\frac{\partial \Omega_{\mathbf{k}}^{0}}{\partial p}\right)_{s}, \mathbf{B}_{\mathbf{k}}=-\left(\mathbf{e}_{z} \times \mathbf{k}\right) \frac{f\left(\mathbf{u}_{s} \cdot \mathbf{k}\right)}{k^{2}} \Re\left(\frac{\partial \Omega_{\mathbf{k}}^{0}}{\partial p}\right)_{s} \\
& \boldsymbol{\tau}_{\mathbf{k}}^{v}(p)=\mathbf{k} \frac{\left(\mathbf{u}_{s} \cdot \mathbf{k}\right)^{2}}{k^{2}} \Im\left(\Omega_{\mathbf{k}}^{v *} \frac{\partial \Omega_{\mathbf{k}}^{v}}{\partial p}\right), \sigma_{\mathbf{k}}^{v}(p)=\left(\mathbf{e}_{z} \times \mathbf{k}\right) \frac{f\left(\mathbf{u}_{s} \cdot \mathbf{k}\right)^{2}}{n_{\mathbf{k}}^{v} k^{2}} \Re\left(\Omega_{\mathbf{k}}^{v *} \frac{\partial \Omega_{\mathbf{k}}^{v}}{\partial p}\right),
\end{aligned}
$$

where $\mathbf{A}_{\mathbf{k}}$ and $\mathbf{B}_{\mathbf{k}}$ represent the spectral amplitudes of the surface drag and transverse drag, whereas $\tau_{\mathbf{k}}^{v}$ and $\sigma_{\mathbf{k}}^{v}$ are the spectral amplitudes of the longitudinal (i.e. parallel to $\mathbf{k}$ ) and transverse (to $\mathbf{k}$ ) stress vectors, respectively.

Note the general relationships valid on the surface due to (3.11c) and the equality $\left(n_{\mathbf{k}}^{v}\left(p_{s}\right)\right)_{v=0}=-\mathbf{u}_{\mathbf{s}} \cdot \mathbf{k}$,

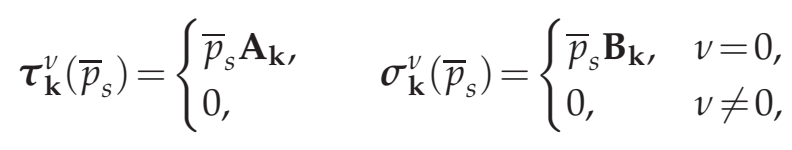

and the formulae following from these relationships in the common coordinates,

$$
\boldsymbol{\tau}\left(\bar{p}_{s}\right)=\bar{p}_{s} \mathbf{A}, \quad \boldsymbol{\sigma}\left(\bar{p}_{s}\right)=\bar{p}_{s} \mathbf{B},
$$

which make sense only in the stationary case. The linear dependence between wave stress and surface drag was first demonstrated by [13, p. 103]. The equations in (3.14) demonstrate that the same is correct for longitudinal and transverse parts of stress and drag in separation. Note that the relationships in (3.14) hold for arbitrary orography and 
atmospheric conditions, being in this respect quite general. However, they do not proceed promptly from the prime definitions in $(2.7 \mathrm{~d})$ and $(2.9 \mathrm{~d})$, but are restricted to linear wave dynamics with a free-slip surface boundary condition because they make use of the linear dependencies (3.4), (3.5) of $\widehat{W}_{\mathbf{k}^{\prime}}^{v} \widehat{\Phi}_{\mathbf{k}^{\prime}}^{v}$ and $\widehat{\Psi}_{\mathbf{k}}^{v}$ on $\widehat{\omega}_{\mathbf{k}}^{v}$ and of the free-slip boundary condition (3.11c). In the case of two-dimensional orography with mountain ridges aligned along the $y$-axis, the stationary mode solution is activated for $\mathbf{k}=k \mathbf{e}_{x}$ only (waves are completely confined to the $x-p$ plane). In this particular situation, the formulae in (3.13) give

$$
\begin{aligned}
& \mathbf{A}_{\mathbf{k}}=\mathbf{e}_{x} A_{k}, \quad \mathbf{B}_{\mathbf{k}}=-\mathbf{e}_{y} B_{k}, \\
& A_{k}=k u_{x s}^{2} \Im\left(\frac{\partial \Omega_{k}^{0}}{\partial p}\right)_{s}, \quad B_{k}=f u_{x s} \Re\left(\frac{\partial \Omega_{k}^{0}}{\partial p}\right)_{s}, \\
& \tau_{\mathbf{k}}^{0}(p)=\mathbf{e}_{x} \tau_{k}^{0}(p), \quad \sigma_{\mathbf{k}}^{0}(p)=-\mathbf{e}_{y} \sigma_{k}^{0}(p), \\
& \tau_{k}^{0}(p)=k u_{x s}^{2} \Im\left(\Omega_{k}^{0 *} \frac{\partial \Omega_{\mathbf{k}}^{0}}{\partial p}\right), \quad \sigma_{k}^{0}(p)=\frac{f u_{x s}^{2}}{u_{x}(p)} \Re\left(\Omega_{k}^{0 *} \frac{\partial \Omega_{k}^{0}}{\partial p}\right) .
\end{aligned}
$$

As it appears from (3.13a), the longitudinal and transverse drag vectors are parallel and transverse to the wave vector $\mathbf{k}$ and thus, they are mutually orthogonal for each separate mode. This orthogonality does not extend back to the stress vectors (3.12a) in Cartesiancoordinates for general 3D flow case. However, the mutual orthogonality of $\tau$ and $\sigma$ will be restored for a two-dimensional flow, as in this particular situation all $\boldsymbol{\tau}_{\left(k_{x}, 0\right)}^{v}$ are collinear with $\mathbf{e}_{x}$, while all $\sigma_{\left(k_{x}, 0\right)}^{v}$ are collinear with $\mathbf{e}_{y}$.

\subsection{Stress conservation}

An absence (nullification) of the drag on some pressure level means conservation of the stress at the same level and can be expressed as the zero vertical convergence condition

$$
\mathbf{c}_{l}=\frac{\partial \tau}{\partial p}=0, \quad \mathbf{c}_{t}=\frac{\partial \sigma}{\partial p}=0,
$$

or equivalently in the orthogonal-mode presentation

$$
\frac{\partial \tau_{\mathbf{k}}^{v}}{\partial p}=0, \quad \frac{\partial \sigma_{\mathbf{k}}^{v}}{\partial p}=0
$$

To our best knowledge, the transverse stress conservation was not investigated (at least in explicit mode) before. Conservation of the longitudinal stress was proved for stationary, two-dimensional zonal flows in [16]. The proof is often named the EliassenPalm theorem. We will investigate, in which extent this theorem can be extend to threedimensional flows with turning reference wind, including transverse stress conservation. 


\subsubsection{Longitudinal stress conservation}

Applying vertical divergence to (3.13b):

$$
\frac{\partial \tau_{\mathbf{k}}^{v}}{\partial p}=\frac{\left(\mathbf{u}_{s} \cdot \mathbf{k}\right)^{2}}{k^{2}} \Im\left(\Omega_{\mathbf{k}}^{v *} \frac{\partial^{2} \Omega_{\mathbf{k}}^{v}}{\partial p^{2}}\right),
$$

and substitution of the second vertical derivative from the wave equation (3.7a) yields

$$
\frac{\partial \tau_{\mathbf{k}}^{v}}{\partial p}=-\frac{\left(\mathbf{u}_{s} \cdot \mathbf{k}\right)^{2}}{k^{2}} \Im\left(\frac{Q_{\mathbf{k}}^{v}}{p} \Omega_{\mathbf{k}}^{v *} \frac{\partial \Omega_{\mathbf{k}}^{v}}{\partial p}+\frac{\Lambda_{\mathbf{k}}^{v}}{p^{2}} \Omega_{\mathbf{k}}^{v *} \Omega_{\mathbf{k}}^{v}\right) .
$$

The right side vanishes, if the imaginary part of the round brackets becomes zero, which happens if $Q_{\mathbf{k}}^{v}$ vanishes and $\Lambda_{\mathbf{k}}^{v}$ becomes real simultaneously:

$$
Q_{\mathbf{k}}^{v}=0, \quad \Im \Lambda_{\mathbf{k}}^{v}=0 .
$$

Closer look on (3.7b), (3.7c) reveals that this occurs, either if

$$
f \rightarrow 0,
$$

or

$$
v_{\mathbf{k}}^{1} \rightarrow 0, \quad v_{\mathbf{k}}^{2} \rightarrow 0, \quad v_{\mathbf{k}}^{3} \rightarrow 0
$$

simultaneously. Condition (3.17b) means a non-rotating planet or an equatorial location of the modelling domain as minimum. Conditions (3.17c) yield

$$
\frac{\partial \mathbf{u}}{\partial p}=0, \quad\left(p \frac{\partial}{\partial p}\right)^{2} \mathbf{u}=0,
$$

which, if they hold for a layer $\left[p_{1}, p_{2}\right]$ (of finite or infinitesimal depth) withdraw to the wind constancy requirement in that layer

$$
\mathbf{u}(p)=\mathbf{U}, \quad p_{1}<p<p_{2} .
$$

This condition presents the only possible situation when the Eliassen-Palm theorem is satisfied precisely (in the given layer) for all longitudinal stress components $\tau_{\mathbf{k}}^{v}$. Note that the orography can be three-dimensional, but the flow must remain two-dimensional in the layer, as no wind rotation with altitude is permitted by $(3.17 \mathrm{e})$ either.

If we confine treatment with the simplified wave equation (3.8), conditions (3.17a) are satisfied per se, and thus, all longitudinal stress components become conservative without restrictions to the wind, temperature or orography conditions (i.e. arbitrary 3D orography spectrum is allowed with the wave vector $\mathbf{k}$ spanning azimuthally $180^{\circ}$ ).

However, in the general case of non-constant wind, the smallness of perturbation terms $Q_{\mathbf{k}}^{v}$ and $\Im \Lambda_{\mathbf{k}}^{v}$ becomes questionable. Quantitatively these two terms can be neglected, if they become small in comparison with the main real part of $\Lambda$ :

$$
\left|Q_{\mathbf{k}}^{v} / \Re \Lambda_{\mathbf{k}}^{v}\right| \ll 1, \quad\left|\Im \Lambda_{\mathbf{k}}^{v} / \Re \Lambda_{\mathbf{k}}^{v}\right| \ll 1,
$$


which readily withdraws to the smallness requirement for non-dimensional parameters

$$
\begin{array}{ll}
\varepsilon_{k}^{1}=\left|\frac{f^{2} v_{\mathbf{k}}^{1}}{(H k N)^{2} n_{\mathbf{k}}^{v}}\right| \sim \frac{h_{k}^{1}}{\Delta z}, \quad h_{k}^{1}=\frac{f^{2}}{H k^{2} N^{2}}, \\
\varepsilon_{k}^{2}=\left|\frac{f v_{\mathbf{k}}^{2}}{(H k N)^{2}}\right| \sim \frac{h_{k}^{2}}{\Delta z}, \quad h_{k}^{2}=\frac{f U}{H k N^{2}}, \\
\varepsilon_{k}^{3}=\left|\frac{f v_{\mathbf{k}}^{3}}{(H k N)^{2}}\right| \sim\left(\frac{h_{k}^{3}}{\Delta z}\right)^{2}, & h_{k}^{3}=\sqrt{\frac{f U}{k N^{2}}},
\end{array}
$$

where $U$ is the wind variation amplitude, $\Delta z$ is the wind vertical variation scale, and $k=$ $|\mathbf{k}|$. Above, estimations are applied

$$
p \frac{\partial}{\partial p} \sim \frac{H}{\Delta z}, \quad n_{\mathbf{k}}^{v} \sim U k, \quad v_{\mathbf{k}}^{1} \sim v_{\mathbf{k}}^{2} \sim k U \frac{H}{\Delta z}, \quad v_{\mathbf{k}}^{3} \sim k U\left(\frac{H}{\Delta z}\right)^{2} .
$$

The corresponding to frequencies $v_{\mathbf{k}}^{i}$ scale heights $h_{k}^{i}$ measure the vertical variability scale $\Delta z$ of the wind. If the wind changes slow with the height, so that $\Delta z \gg h_{k}^{i}$, the corresponding $\varepsilon_{k}^{i} \rightarrow 0$, the corresponding characteristic frequency $v_{\mathbf{k}}^{i} \rightarrow 0$ can be neglected in the perturbation terms $Q_{\mathbf{k}}^{v}$ and $\Im \Lambda_{\mathbf{k}^{\prime}}^{v}$ and the longitudinal stress becomes conservative. Vice versa, for $\Delta z \sim h_{k}^{i}$ the perturbation terms become large and the stress becomes convergent. Note that the parameters $\varepsilon_{k}^{i}$ are correct defined and their smallness will be sufficient for stress conservation in any vicinity of the critical levels $p_{c}$ (defined by the condition $\left.n_{\mathbf{k}}^{v}\left(p_{c}\right)= \pm f\right)$ except the critical levels themselves, where $\Lambda_{\mathbf{k}}^{v}, Q_{\mathbf{k}}^{v} \rightarrow \infty$ in separation. As the waves are absorbed (i.e. will vanish) on their critical levels, this will lead to the discontinuity of the longitudinal stress at the passage of critical levels: being a finite constant below of the critical level, the stress amplitude must become abrupt zero above it. As numerical simulations will show, this is the case indeed.

In Table $1, h_{k}^{i}$ are presented for long $\left(k=0.01 \mathrm{~km}^{-1}\right)$, medium $\left(k=0.1 \mathrm{~km}^{-1}\right)$, and short $\left(k=0.3-1 \mathrm{~km}^{-1}\right)$ parts of the wave spectrum. As seen, the scale height $h_{k}^{3}$ is much larger of other two in the whole spectral domain of $k$, being the only large enough able to cause substantial $\varepsilon_{k}^{3}$ and stress convergence for wind variability scales $\Delta z \sim 100-1000 \mathrm{~m}$. Such variability scales are typical for the planetary boundary layer and adjacent lower troposphere, though rapid direction changes are observable in fronts and cyclones in the upper troposphere also. The large $h_{k}^{3}$ points to substantial $v_{\mathbf{k}}^{3}$. A look on $v_{\mathbf{k}}^{3}$ (3.7f) shows

Table 1: Scale heights $h_{k}^{i}$ at different wavenumbers $k$.

\begin{tabular}{||ll|c|c|c|c||}
\hline$k$, & $\mathrm{km}^{-1}$ & 0.01 & 0.1 & 0.3 & 1 \\
\hline$h_{k}^{1}$, & $\mathrm{m}$ & 100 & 1 & 0.1 & 0.01 \\
$h_{k}^{2}$, & $\mathrm{m}$ & 100 & 10 & 3 & 1 \\
$h_{k}^{3}$, & $\mathrm{m}$ & 1000 & 550 & 180 & 100 \\
\hline
\end{tabular}


that the large variability scale $h_{k}^{3}$ results from large curvature of wind (i.e., from large $\left.(p \partial / \partial p)^{2} \mathbf{u}\right)$, which can be reached through rapid wind changes either in the amplitude or in the direction. Wind rotation is not required in the general 3D case, i.e. the stress becomes variable even if the wind is not rotating but just sheared vertically. That means by the way that the Eliassen-Palm theorem can not be extended to the 3D-orography case even for a non-rotating wind. An important exception is however presented at the stationary wave generation by 2D orography, as in this special case the wave-spectrum is one-dimensional, confined to $\mathbf{k}=k_{x} \mathbf{e}_{x}$. According to (3.6b), (3.7f), we will have $v_{\mathbf{k}}^{3}=$ $k_{x}(p \partial / \partial p)^{2} u_{y}$, which turns zero either if wind is not returning and directed along $x$ axis, or the parallel to mountain crest wind component $u_{y}$ remains constant. That is we return to the classical Eliassen-Palm result for two dimensional non-rotating flow and two-dimensional orography.

\subsubsection{Transverse stress conservation}

Analogical investigation of the transverse stress conservation with wave equation solution attraction shows that the isothermal 2D flow with constant wind $\mathbf{U}$ presents the single case, when $\sigma_{k}^{0}$ becomes constant with height in the free wave domain. In the general variable temperature case, small parameters analogous to $\varepsilon_{k}^{i}$ are absent and variations of $\sigma_{\mathbf{k}}^{v}(p)$ with height are co-measurable with the wind variations.

In summary, we can say that the longitudinal stress is conserved in the layers where the wind remains constant, and also in the case of two-dimensional orography for the wind, transverse to the mountain crest and changing with the altitude without rotation. It is never conserved in the layers with rotating wind, though convergence can be small for $\Delta z \ll h_{k}^{3}$, i.e. for $\varepsilon_{k}^{3} \rightarrow 0$. The transverse drag is always convergent. In more detail these properties will be investigated further at the numerical modelling.

\subsubsection{Relation with the theory of linear momentum fluxes}

An attempt to generalise Palm-Eliassen theorem to the 3D flow conditions was made by Broad [49] in frames of the linear momentum fluxes theory. Broad introduced a new kind of stress (named the Broad stress hereafter), and reported its conservation for general 3D reference flow case. As this result is fundamentally different from which was established above for the longitudinal and transverse stress, the subject requires addressing in frames of the present spectral theory. In our notation, the Broad stress reads

$$
\widetilde{\gamma}=\overline{\omega\left(\mathbf{v}^{\prime}+f \mathbf{e}_{\mathbf{z}} \times \delta \mathbf{x}\right)}=\gamma+f \mathbf{e}_{z} \times \overline{\omega \delta \mathbf{x}}=\sum_{\mathbf{k}} \omega_{\mathbf{k}}^{0 *}\left(\mathbf{v}_{\mathbf{k}}^{\prime 0}+f \mathbf{e}_{z} \times \delta \mathbf{x}_{\mathbf{k}}^{0}\right) .
$$

The special notation $\widetilde{\gamma}$ is introduced by us, also the transition from the original $z$-coordinates to the isobaric coordinate system is made, using substitution $\rho w=-\omega / g \rightarrow \omega$. The Broad stress, which is specified for stationary waves, includes (in addition to the common wave stress (2.7b)) a supplementary vector proportional to the horizontal displacement 
field $\delta \mathbf{x}$ defined in [49] via $\mathbf{v}^{\prime}=d_{0} \delta \mathbf{x} / d t$, yielding $\mathbf{v}_{\mathbf{k}}^{\prime 0}=\mathrm{i} n_{\mathbf{k}}^{0} \delta \mathbf{x}_{\mathbf{k}}^{0}=-\mathrm{i} v_{\mathbf{k}}^{0} \delta \mathbf{x}_{\mathbf{k}}^{0}$. Using this relationship for $\delta \mathbf{x}_{\mathbf{k}}^{0}$ expression, $\widetilde{\gamma}$ becomes

$$
\widetilde{\gamma}=\sum_{\mathbf{k}} \omega_{\mathbf{k}}^{0 *}\left(\mathbf{v}_{\mathbf{k}}^{\prime 0}+\mathrm{i} \frac{f}{v_{\mathbf{k}}^{0}} \mathbf{e}_{z} \times \mathbf{v}_{\mathbf{k}}^{\prime 0}\right)
$$

The further result will depend on the velocity perturbation $\mathbf{v}_{\mathbf{k}}^{\prime 0}$ choice. In the case of the full perturbation with both potential and rotational components included in (3.4a), application of (3.4c), (3.4d) and (3.5b) yields

$$
\mathbf{v}^{\prime 0}{ }_{\mathbf{k}}=-\frac{\mathrm{i} \mathbf{k}}{k^{2}} \frac{\partial \widehat{\omega}_{\mathbf{k}}^{0}}{\partial p}-\frac{\left(\mathbf{e}_{z} \times \mathbf{k}\right)}{k^{2}}\left(\frac{f}{v_{\mathbf{k}}^{0}} \frac{\partial \widehat{\omega}_{\mathbf{k}}^{0}}{\partial p}+\mathrm{i} \frac{v_{\mathbf{k}}^{2}(p)}{v_{\mathbf{k}}^{0}} \frac{\widehat{\omega}_{\mathbf{k}}^{0}}{p}\right)
$$

enabling to present (3.18a) as

$$
\widetilde{\gamma}=\widetilde{\tau}+2 \sigma .
$$

Here $\sigma$ is the stationary transverse drag presented with the first sum ( $v=0$ case) in (3.12c), while the longitudinal component

$$
\widetilde{\boldsymbol{\tau}}=\sum_{\mathbf{k}}\left(1+\frac{f^{2}}{(\mathbf{k} \cdot \mathbf{u})^{2}}\right) \tau_{\mathbf{k}}^{0}\left|\mu_{\mathbf{k}}\right|^{2}
$$

(where $\tau_{\mathbf{k}}^{0}$ is presented in (3.13b)) withdraws to the common longitudinal stress (3.12b), only if the orography spectrum is located in the spectral domain $f^{2} /(\mathbf{k} \cdot \mathbf{u})^{2} \ll 1$. The presence of factor 2 in front of $\sigma$ and additional term in the round brackets in (3.18c) proceed from the double implication of the rotational part of velocity: first in the sum (3.18a) and second in the (3.18b). Indeed, if the non-rotational velocity fluctuation

$$
\mathbf{v}_{\mathbf{k}}^{\prime 0}=-\frac{i \mathbf{k}}{k^{2}} \frac{\partial \widehat{\omega}_{\mathbf{k}}^{0}}{\partial p}
$$

is assumed in (3.18a), then

$$
\widetilde{\gamma}=\tau+\sigma
$$

coincidentally with our stress definition. Though there is no explicit hint in [49] of which wind perturbation was assumed, the usage of non-simplified 3D linear equations points rather to (3.18b). Universal conservation of $\widetilde{\gamma}$ for arbitrary 3D orography and reference wind distribution was disproved for (3.18f) above. It can be disproved in the similar way for (3.18c), i.e. acting on (3.18c) with $d / d p$, and using the wave equation on the right side to eliminate the second derivatives of $\Omega_{\mathbf{k}}^{0}$ where appropriate. Thus, the general conclusion can be drawn that the Broad stress is not conservative in arbitrary 3D orography and reference wind condition; its involvement will not extend the Palm-Eliassen theorem from 2D zonal lows to more complex flow conditions. 


\subsection{Numerical realisation of the wave model}

Basics of the NWM are presented in RZ07. Some further refinement in the vertical discretisation and radiation boundary condition treatment is introduced meanwhile which are shortly discussed in following, confining the treatment to stationary case $v=0$.

The wave equation is discretised horizontally with the grid steps $\Delta x=2 L_{x} / 2 N_{x}, \Delta y=$ $2 L_{y} / 2 N_{y}$. Typically $\Delta x=\Delta y=500 \mathrm{~m}, N_{x}=N_{y}=512$, which yields the area size $2 L_{x}=2 L_{y}=$ $512 \mathrm{~km}$. The stationary part of the Fourier sum in (3.3a) is replaced with a $2 \mathrm{D}$ discrete Fourier series

$$
\begin{aligned}
& \omega\left(\mathbf{x}_{\mathbf{j}}, p_{l}\right)=\omega\left(j_{x} \Delta x, j_{y} \Delta y, p_{l}\right) \equiv \omega_{\mathbf{j}, l} \\
= & \sum_{j_{x}^{\prime}=-N_{x}+1}^{N_{x}-1} \sum_{j_{y}^{\prime}=-N_{y}+1}^{N_{y}-1} \widehat{\omega}_{\mathbf{j}^{\prime} l} \mathrm{e}^{-\mathrm{i}\left(\mathbf{x}_{\mathbf{j}} \cdot \mathbf{k}_{\mathbf{j}^{\prime}}\right)}=\sum_{j_{x}^{\prime}=-N_{x}+1 j_{y}^{\prime}=-N_{y}+1}^{N_{x}-1} \sum_{\widehat{\omega}_{j l}}^{N_{y}-1} \mathrm{e}^{-\mathrm{i} \pi\left(j_{x} j_{x}^{\prime} / N_{x}+j_{y} j_{y}^{\prime} / N_{y}\right)} .
\end{aligned}
$$

The components of horizontal wave-vector $k_{j x}=j_{x} \pi / L_{x}, k_{j y}=j_{y} \pi / L_{y}$ maintain their previous sense (see (3.3c)), except their numbers become finite, equal to $2 N_{x}-1$ and $2 N_{y}-1$ consequently. However, proceeding from the gradient $\nabla=\mathbf{e}_{x} \frac{\partial}{\partial x}+\mathbf{e}_{x} \frac{\partial}{\partial y}$ and Laplacian $\nabla^{2}$ to their discrete analogues $\widetilde{\nabla}=\mathbf{e}_{x} \widetilde{\nabla}_{x}+\mathbf{e}_{x} \widetilde{\nabla}_{y}, \widetilde{\nabla}^{2}=\widetilde{\nabla}_{x}^{2}+\widetilde{\nabla}_{y}^{2}$, where

$$
\begin{aligned}
& \widetilde{\nabla}_{x} \omega_{i j}=\left(\omega_{i+1, j}-\omega_{i-1, j}\right) /(2 \Delta x), \\
& \widetilde{\nabla}_{y} \omega_{i j}=\left(\omega_{i, j+1}-\omega_{i, j-1}\right) /(2 \Delta y), \\
& \widetilde{\nabla}_{x}^{2} \omega_{i j}=\left(\omega_{i+1, j}+\omega_{i-1, j}-2 \omega_{i j}\right) / \Delta x^{2}, \\
& \widetilde{\nabla}_{y}^{2} \omega_{i j}=\left(\omega_{i, j+1}+\omega_{i, j-1}-2 \omega_{i j}\right) / \Delta y^{2},
\end{aligned}
$$

yields for (3.19a) replacement of the continuous Fourier images of differential operators by consequent discrete images as follows

$$
\begin{aligned}
& \frac{\partial}{\partial x} \sim-\mathrm{i} k_{x} \rightarrow \widetilde{\nabla}_{x} \sim-\mathrm{i} \widetilde{k}_{j x}=-\mathrm{i} \frac{\sin \left(j_{x} \pi / N_{x}\right)}{\Delta x}, \\
& \frac{\partial}{\partial y} \sim-\mathrm{i} k_{y} \rightarrow \widetilde{\nabla}_{y} \sim-\mathrm{i} \widetilde{k}_{j y}=-\mathrm{i} \frac{\sin \left(j_{y} \pi / N_{y}\right)}{\Delta y}, \\
& \frac{\partial^{2}}{\partial x^{2}} \sim-k_{x}^{2} \rightarrow \widetilde{\nabla}_{x}^{2} \sim-\widetilde{k}_{j x}^{2}=-\frac{4 \sin ^{2}\left(j_{x} \pi / N_{x}\right)}{\Delta x^{2}}, \\
& \frac{\partial^{2}}{\partial y^{2}} \sim-k_{y}^{2} \rightarrow \widetilde{\nabla}_{y}^{2} \sim-\widetilde{k}_{j y}^{2}=-\frac{4 \sin ^{2}\left(j_{y} \pi / N_{y}\right)}{\Delta y^{2}} .
\end{aligned}
$$

The horizontal discretisation yields replacement of $k_{x}, k_{y}, k_{x}^{2}, k_{y}^{2}$ to the consequent discrete Fourier images $\widetilde{k}_{x}, \widetilde{k}_{y}, \widetilde{k}_{x}^{2}, \widetilde{k}_{y}^{2}$ everywhere in formulae (3.4) through (3.18). 
The vertical main grid $p_{l}$, the auxiliary half-level grid $p_{l+1 / 2}$ and the consequent absolute and relative pressure differences are

$$
\begin{aligned}
& 0<p_{0}<p_{1}<p_{2}<\cdots<p_{l-1}<p_{l}<\cdots<p_{N_{p}-1}<p_{N_{p}}<p_{N_{p}+1}=\overline{p_{s}} \\
& p_{l+1 / 2}=\frac{p_{l}+p_{l+1}}{2}, \quad \Delta p_{l+1 / 2}=p_{l+1}-p_{l}, \quad \Delta p_{l}=p_{l+1 / 2}-p_{l-1 / 2} \\
& \delta_{l}=\frac{\Delta p_{l}}{p_{l}}, \quad \delta_{l+1 / 2}=\frac{\Delta p_{l+1 / 2}}{p_{l+1 / 2}} .
\end{aligned}
$$

The main grid is specified from numerical solution of the barometric equation

$$
\frac{\partial p}{\partial z}=-\frac{p}{H} \rightarrow \frac{p_{l}-p_{l-1}}{\Delta z_{l-1 / 2}}=\frac{p_{l}+p_{l-1}}{H_{l}+H_{l-1}} \rightarrow p_{l-1}=p_{l} \frac{H_{l}+H_{l-1}-\Delta z_{l-1 / 2}}{H_{l}+H_{l-1}+\Delta z_{l-1 / 2}} .
$$

The the vertical resolution and extent of modelling domain are controlled by a variable set of vertical Cartesian-coordinate grid-steps $\Delta z_{l-1 / 2}=z_{l}-z_{l-1}$. A general limitation is set

$$
\frac{\Delta z_{l-1 / 2}}{H_{l-1 / 2}}=\frac{p_{l}-p_{l-1}}{p_{l}+p_{l-1}}=2 \frac{\Delta p_{l-1 / 2}}{p_{l-1 / 2}}=2 \delta_{l-1 / 2} \leq 10^{-2},
$$

which provides sufficient resolution for all levels with the reference wind $|\mathbf{U}|>1 \mathrm{~m} / \mathrm{s}$. Most typically they are constant $\Delta z_{l-1 / 2}=100 \mathrm{~m}$, which for the top boundary level $p_{0}$ at 30 $\mathrm{km}$ altitude will yield $N_{p}=300$. In the neighbourhood of critical levels where $|\mathbf{U}|$ becomes small, the resolution is increased up to $\Delta z_{l-1 / 2}=10 \mathrm{~m}$. Such high resolution is also applied in some cases at accuracy tests throughout the atmosphere, yielding $N_{p} \sim 3000$.

For each $\mathbf{j}=\left\{j_{x}, j_{y}\right\}$, Eq. (3.7a) is discretised to a second order difference equation in the form of a linear algebraic system

$$
\begin{aligned}
& M_{0}^{-} \widehat{\omega}_{\mathbf{j} 0}+M_{1}^{0} \widehat{\omega}_{\mathbf{j} 1}+M_{2}^{+} \widehat{\omega}_{\mathbf{j} 2}=0, \quad l=1, \\
& M_{l}^{-} \widehat{\omega}_{\mathbf{j} l-1}+M_{l}^{0} \widehat{\omega}_{\mathbf{j} l}+M_{l}^{+} \widehat{\omega}_{\mathbf{j} l+1}=0, \quad l=2, \cdots, N_{p}-1, \\
& M_{N_{p}}^{-} \widehat{\omega}_{\mathbf{j} N_{p}-1}+M_{N_{p}}^{0} \widehat{\omega}_{\mathbf{j} N_{p}}+M_{N_{p}}^{+} \widehat{\omega}_{\mathbf{j} N_{p}+1}=0, \quad l=N_{p},
\end{aligned}
$$

with matrix elements (depending also on the horizontal mode-numbers $\mathbf{j}$ via $Q$ and $\Lambda$ )

$$
\begin{aligned}
& M_{l}^{-}=\frac{\delta_{l}}{\delta_{l-1 / 2}}+\frac{\delta_{l}}{2}\left(Q_{\mathbf{j} l}+1\right), \quad M_{l}^{+}=\frac{\delta_{l}}{\delta_{l+1 / 2}}-\frac{\delta_{l}}{2}\left(Q_{\mathbf{j} l}+1\right), \\
& M_{l}^{0}=\delta_{l}^{2} \Lambda_{\mathbf{j} l}-\left[\frac{\delta_{l}}{\delta_{l+1 / 2}}+\frac{\delta_{l}}{\delta_{l-1 / 2}}\right],
\end{aligned}
$$

System $(3.19 \mathrm{k})-(3.19 \mathrm{~m})$ consists of $N_{p}$ equations for $N_{p}$ unknown complex quantities $\widetilde{\omega}_{l}$, specified at the internal pressure levels $p_{1}, \cdots, p_{N_{p}}$. The first $l=1$ and last $l=N_{p}$ equations in $(3.19 \mathrm{k})-(3.19 \mathrm{~m})$ include the spectral amplitude at the top boundary, $\widehat{\omega}_{\mathrm{j} 0}$, and at the surface, $\widehat{\omega}_{\mathbf{j}} N_{p}+1$, which have to be specified from appropriate boundary conditions. At 
the surface boundary $p_{N_{p}+1}=\overline{p_{s}}$, the free-slip condition (3.11b) is applied, which in the discrete case becomes

$$
\widehat{\omega}_{\mathbf{j} N_{p}+1}=-\mathrm{i}\left(\mathbf{U}_{N_{p}+1} \cdot \widetilde{\mathbf{k}}_{\mathbf{j}}\right) \bar{p}_{s} \widehat{\mu}_{\mathbf{j}} .
$$

It would be misleading to apply the rigid-lid condition $\widehat{\omega}_{\mathrm{j} 0}=0$ at the top, as this would produce strong spurious top reflection of buoyancy waves. Rather, the radiation boundary condition [50] must be applied, which yields

$$
\widehat{\omega}_{\mathbf{j} 0}=C_{\mathbf{j}} \widehat{\omega}_{\mathbf{j} 1},
$$

where constants $C_{\mathbf{j}}$ depend beside mode-numbers $\mathbf{j}$ on the atmospheric background parameters at the top, $\mathbf{U}_{0}=\mathbf{U}\left(p_{0}\right), H_{0}=H\left(p_{0}\right)$ and $N_{0}=N\left(p_{0}\right)$. System (3.19k)-(3.19m) presents then as

$$
\begin{aligned}
& \left(M_{0}^{-} C_{\mathbf{j}}+M_{1}^{0}\right) \widehat{\omega}_{\mathbf{j} 1}+M_{2}^{+} \widehat{\omega}_{\mathbf{j} 2}=0, \quad l=1, \\
& M_{l}^{-} \widehat{\omega}_{\mathbf{j} l-1}+M_{l}^{0} \widehat{\omega}_{\mathbf{j} l}+M_{l}^{+} \widehat{\omega}_{\mathbf{j} l+1}=0, \quad l=2, \cdots, N_{p}-1, \\
& M_{N_{p}}^{-} \widehat{\omega}_{\mathbf{j} N_{p}-1}+M_{N_{p}}^{0} \widehat{\omega}_{\mathbf{j} N_{p}}=-M_{N_{p}}^{+} \widehat{\omega}_{\mathbf{j} N_{p}+1}, \quad l=N_{p} .
\end{aligned}
$$

Initially in RZ07 the radiation condition was introduced and $C_{\mathbf{j}}$ were determined assuming that in the upper boundary vicinity, the wanted solution $\widehat{\omega}_{\mathrm{j} l}$ coincides (within a constant multiplier precision) with a wave $\widetilde{\omega}_{\mathrm{j} l}$ which transports energy upward (and has a phase retard with the altitude) in a homogeneous medium with constant $\mathbf{U}=\mathbf{U}_{0}, H=H_{0}$ and $N=N_{0}$. Such wave satisfies equation (3.7a) with $Q=0, \Lambda=\Lambda_{\mathbf{j}}\left(p_{0}\right)=\Lambda_{\mathbf{j}}^{0}$, and presents analytically

$$
\widetilde{\omega}_{\mathbf{j}}(p)=\left(\frac{p}{p_{*}}\right)^{1 / 2+\mathrm{i} m_{\mathbf{j}}}, \quad m_{\mathbf{j}}=\sqrt{\Lambda_{\mathbf{j}}^{0}-1 / 4},
$$

where $p_{*}$ is a fixed reference level near the top. In RZ07, $p_{*}=p_{1 / 2}$ was chosen, which gives

$$
\widetilde{\omega}_{\mathbf{j} 0}=\left(\frac{p_{0}}{p_{1 / 2}}\right)^{1 / 2+\mathrm{i} m_{\mathrm{j}}}, \widetilde{\omega}_{\mathbf{j} 1}=\left(\frac{p_{1}}{p_{1 / 2}}\right)^{1 / 2+\mathrm{i} m_{\mathrm{j}}} \rightarrow C_{\mathbf{j}}=\frac{\widetilde{\omega}_{\mathbf{j} 0}}{\widehat{\omega}_{\mathrm{j} 1}}=\left(\frac{p_{1 / 2}-\Delta p_{1 / 2}}{p_{1 / 2}+\Delta p_{1 / 2}}\right)^{1 / 2+\mathrm{i} m_{\mathrm{j}}} .
$$

Another option to specify $C_{\mathrm{j}}$ is to use the differential radiation boundary condition (DRBC) which was first applied in a vertically discrete wave equation by [51]. DBRC proceeds from $(3.19 \mathrm{u})$ in both continuous and discrete forms as

$$
\left[p \frac{\partial \widehat{\omega}}{\partial p}-\left(1 / 2+\mathrm{i} m_{\mathbf{j}}\right) \widehat{\omega}\right]_{p=p_{1 / 2}}=0 \rightarrow p_{1 / 2} \frac{\widehat{\omega}_{1}-\widehat{\omega}_{0}}{\Delta p_{1 / 2}}-\left(1 / 2+\mathrm{i} m_{\mathbf{j}}\right) \frac{\widehat{\omega}_{1}+\widehat{\omega}_{0}}{2}=0,
$$

yielding

$$
C_{\mathbf{j}}=\frac{\widetilde{\omega}_{\mathbf{j} 0}}{\widehat{\omega}_{\mathbf{j} 1}}=\frac{1-q_{\mathbf{j}}}{1+q_{\mathbf{j}}} \widehat{\omega}_{\mathbf{j} 1}, \quad q_{\mathbf{j}}=\left(1 / 2+\mathrm{i} m_{\mathbf{j}}\right) \frac{p_{1}-p_{0}}{p_{1}+p_{0}} .
$$


However, the right finite-difference condition in $(3.19 \mathrm{w})$ is valid only for small

$$
\frac{\Delta p_{1 / 2}}{p_{1 / 2}}=2 \frac{p_{1}-p_{0}}{p_{1}+p_{0}} \ll 1,
$$

in which case the defined in $(3.19 x) C_{j}$ will coincide with the linearised with respect to $\Delta p_{1 / 2}$ version of $C_{\mathbf{j}}$ in $(3.19 \mathrm{v})$. Numerical experimentation reveals that $(3.19 \mathrm{y})$ presents an essential precondition for solution accuracy. In the present NWM this condition is fulfilled due to the global condition (3.19j).

System (3.19r)-(3.19t) can be solved as an initial value problem or a boundary value problem for one-dimensional Laplace (i.e. second order elliptical homogeneous) equation (3.7a). In the first case (applied in RZ07) a provisional initial value 1 is assigned to the topmost amplitude $\widehat{\omega}_{\mathbf{j} 1}=\widetilde{\omega}_{1}=1$, after which the remaining provisional amplitudes $\widetilde{\omega}_{l}$ are solved (integrated) from (3.19r)-(3.19t) step by step, including the surface value $\widetilde{\omega}_{N_{p}+1}$. The final solution is then re-normed from the provisional one using the lower boundary condition (3.19p)

$$
\widehat{\omega}_{\mathbf{j} l}=\left[-\mathbf{i}\left(\mathbf{U}_{N_{p}+1} \cdot \widetilde{\mathbf{k}}_{\mathbf{j}}\right) \bar{p}_{s} \widehat{\mu}_{\mathbf{j}}\right] \frac{\widetilde{\omega}_{l}}{\widetilde{\omega}_{N_{p}+1}} .
$$

In the second case, system (3.19r)-(3.19t) is treated as a linear non-homogeneous $N_{p^{-}}$ dimensional algebraic system with the three-diagonal matrix and known right side vector (with all components except the last one equal to zero), which is solved numerically using the standard Gaussian elimination method.

These two methods appear algebraically equivalent. The numerical testing shows that they have similar computational efficiency also.

\section{Application of the NWM}

\subsection{Background state of the atmosphere}

A cold front is chosen to represent the background state of atmosphere. Frontal sounding is used because of the strong winds that change direction with altitude and the low stability in the upper troposphere, supporting intensive wave generation and substantial drag effects. The temperature and wind profiles are extracted from a particular HIRLAM [52] forecast. The sounding profiles of temperature, Brunt-Vaisala frequency, and wind are presented in Fig. 2.

Two profiles of the Brunt-Vaisala frequency are applied: the authentic sounding $N$ (black line in Fig. 2) and the smoothed approximation $N_{1}$ (red line), which accounts for the large zigzag of $N$ but ignores the fine-scale small-amplitude fluctuations. The wind is represented by five different profiles, as described in Table 2 and shown in Fig. 2. The actual sounding with the boundary layer and free-atmosphere shear and rotation is presented as WM6. Other profiles are unidirectional approximations of the real frontal wind 

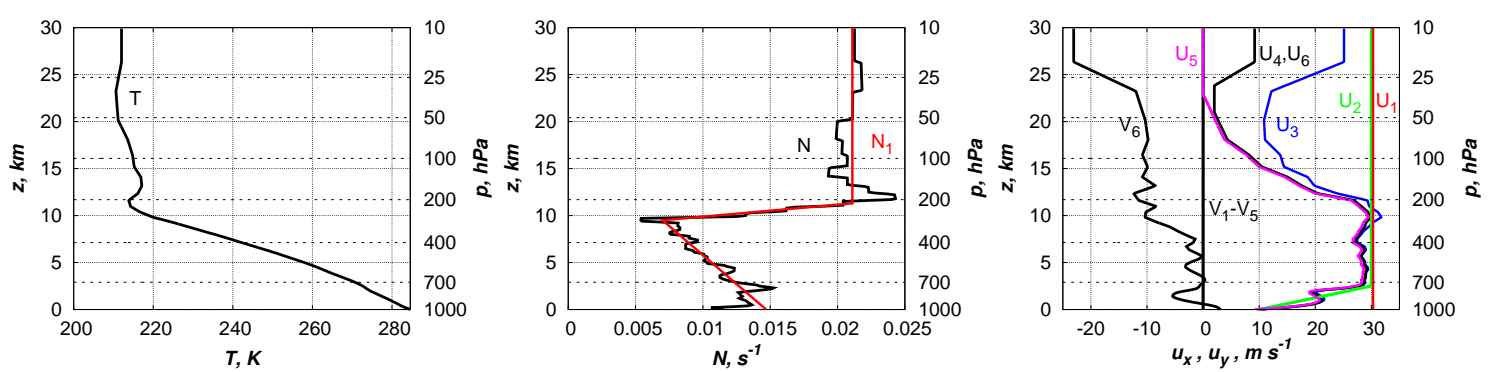

Figure 2: Vertical distribution of the temperature $T$, Brunt-Vaisala frequency $N\left(N_{1}\right.$ is the smoothed approximation of the real buoyancy frequency), and wind components $u_{x}, u_{y}$ for different wind profiles as described in Table 2.

Table 2: Wind models.

\begin{tabular}{||l|c|c|l||}
\hline Model & $u_{x}$ & $u_{y}$ & Comment \\
\hline WM1 & $U_{1}$ & 0 & unidirectional, constant at $30 \mathrm{~m} \mathrm{~s}^{-1}$ wind \\
WM2 & $U_{2}$ & 0 & $\begin{array}{l}\text { unidirectional, linear shear in boundary layer, constant in free } \\
\text { atmosphere }\end{array}$ \\
WM3 & $U_{3}$ & 0 & $\begin{array}{l}U_{3}=|\mathbf{u}|-\text { absolute wind in the front } \\
\text { WM4 }\end{array}$ \\
$U_{4}$ & 0 & unidirectional with frontal $u_{x}$ \\
WM5 & $U_{5}$ & 0 & frontal $u_{x}$ below $22 \mathrm{~km}$, decays to zero at $23.2 \mathrm{~km}$ altitude \\
WM6 & $U_{6}$ & $V_{6}$ & actual frontal wind with rotation \\
\hline
\end{tabular}

in the main frontal wind direction, which is considered as being the wind direction in the lower troposphere at the $p=850 \mathrm{hPa}$ level and taken for the $x$-axis.

The particular front, however, is not tied to any specific orography because the drag and stress spectra (3.13), which are the focus of our investigation, are independent of orographic particularities. Instead, a straight two-dimensional mountain ridge with an Agnesi profile (or a chain of such mountain ridges) is assumed such that it is oriented transverse to the main wind direction ( $x$-axis). The mountain crest is then oriented along the $y$-axis. Such orientation is chosen to achieve the maximum wave-generation intensity in otherwise similar conditions. Stationary orographic waves over a straight ridge always have a two-dimensional structure with wave vectors transverse to the crest, i.e. only stationary modes $\Omega_{\left(k_{x}, 0\right)}^{0}$ with $k_{y}=0$ are activated, despite the wind turns with altitude (the actual wind case WM6) and has a non-zero $y$-component at all levels.

\subsection{Frontal buoyancy waves}

Wave generation in frontal conditions is interesting as a case of trapped wave formation and down-slope windstorm development. Trapped waves arrive on the lee side of mountains, as first described by [48] and modelled thereafter by [21, 25, 53-57]. To illustrate which types of trapped waves are created by various orography in NWM experiments, the wave modelling results are shown for a witch of Agnesi mountain with $h^{0}=2 \mathrm{~km}$ 


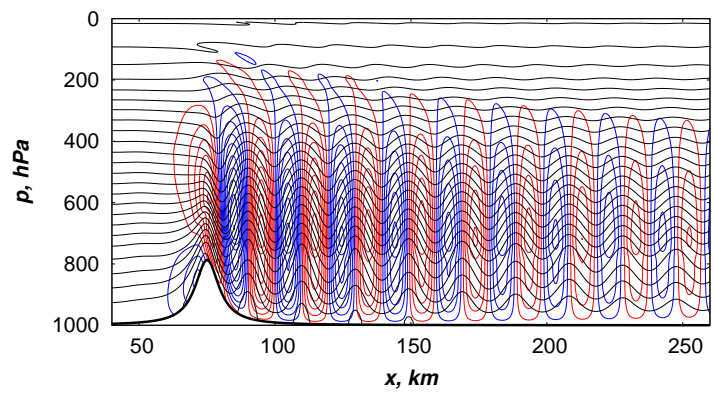

(a) $\Delta \omega=5 \mathrm{Pas}^{-1}, \Delta \Psi^{v} / \bar{p}_{\mathrm{s}}=1 \mathrm{~ms}^{-1}$

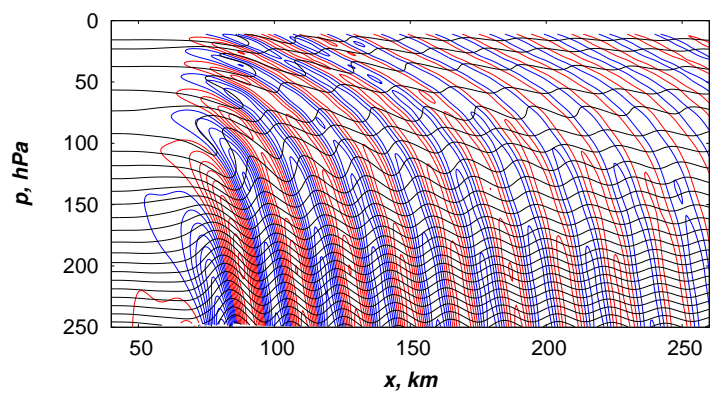

(b) Stratosphere. $\Delta \omega=5 \mathrm{~Pa} \mathrm{~s}^{-1}, \Delta \Psi^{v} / \bar{p}_{\mathrm{s}}=0.1 \mathrm{~m} \mathrm{~s}^{-1}$

Figure 3: Waves in the front with temperature $T$, buoyancy frequency $N$ from Fig. 2, and wind WM3 above and downstream of the bell-shape mountain with $l=5 \mathrm{~km}, h^{0}=2 \mathrm{~km}$. Red and blue isolines represent $\omega>0$ and $\omega<0$, respectively. Thin black curves are the streamlines. (a) entire vertical extent of the atmosphere; (b) stratosphere between 10 and $250 \mathrm{hPa}$ levels.

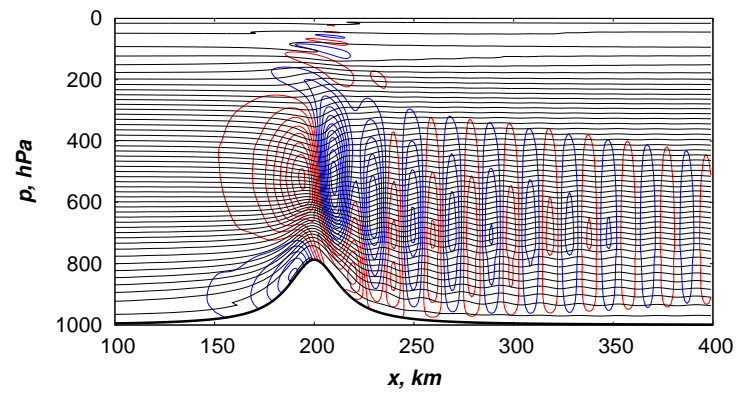

(a) $\Delta \omega=2 \mathrm{~Pa} \mathrm{~s}^{-1}, \Delta \Psi^{v} / \bar{p}_{\mathrm{s}}=0.5 \mathrm{~ms}^{-1}$

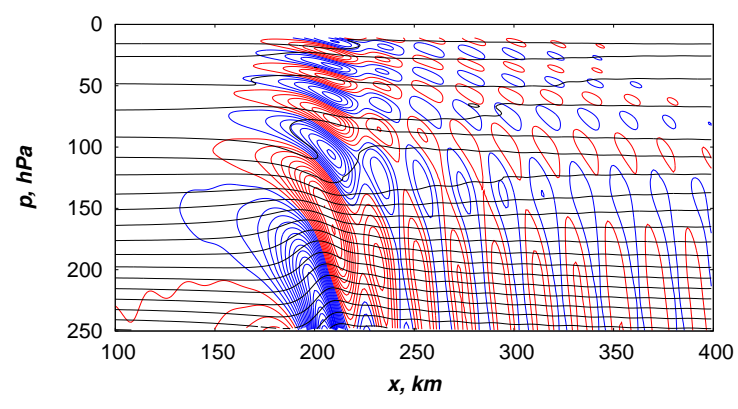

(b) $\Delta \omega=0.25 \mathrm{~Pa} \mathrm{~s}^{-1}, \Delta \Psi^{v} / \bar{p}_{\mathrm{S}}=0.25 \mathrm{~m} \mathrm{~s}^{-1}$

Figure 4: As in Fig. 3, for $l=15 \mathrm{~km}$.

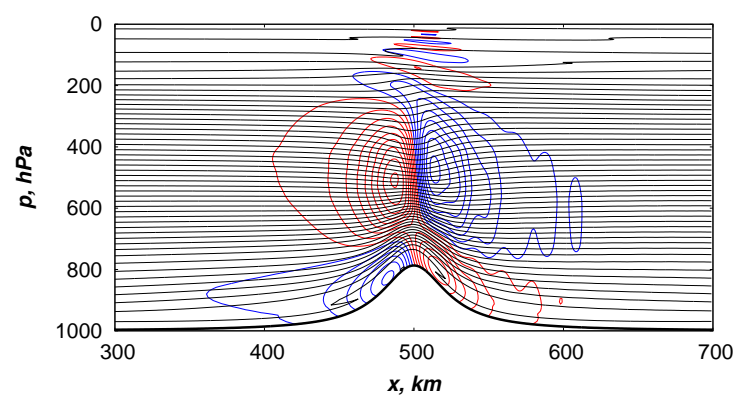

(a) $\Delta \omega=1 \mathrm{Pas}^{-1}, \Delta \Psi^{v} / \bar{p}_{\mathrm{s}}=0.5 \mathrm{~m} \mathrm{~s}^{-1}$

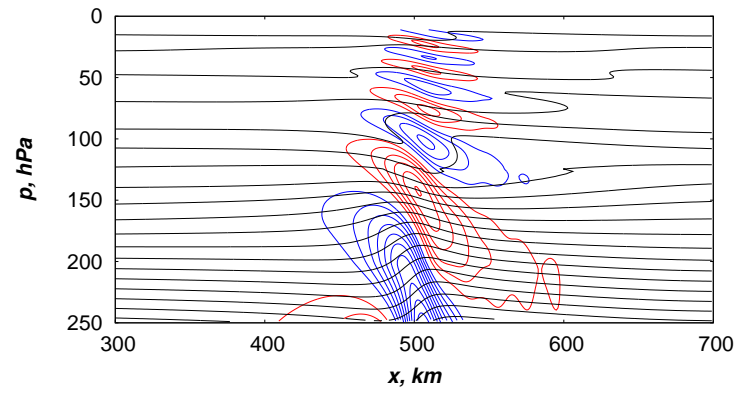

(b) $\Delta \omega=0.25 \mathrm{~Pa} \mathrm{~s}^{-1}, \Delta \Psi^{v} / \bar{p}_{\mathrm{s}}=0.25 \mathrm{~ms}^{-1}$

Figure 5: As in Fig. 3, for $l=25 \mathrm{~km}$.

and $l=5 \mathrm{~km}, 15 \mathrm{~km}$, and $25 \mathrm{~km}$ in Figs. 3 through 5. The modelling in these examples is conducted for real frontal temperature $T$ and buoyancy frequency $N$, as shown in Fig. 2, for the unidirectional wind profile WM3.

To show the potential of using the buoyancy wave equation, the solution $\omega(x, p)$ of 
this equation is first projected into the terrain-following frame of reference (so-called hybrid co-ordinate system), after which the streamlines are calculated as air-particle trajectories in the given stationary wind field $u(p)+u^{\prime}(x, p)=\partial \Psi^{v} / \partial p, \omega(x, p)=-\partial \Psi^{v} / \partial x$, where the velocity fluctuation $u^{\prime}$ corresponds with the modelled $\omega(x, p)$ distribution, while $\Psi^{v}(x, p)$ represents the 'vertical' stream function. These figures show both the $\omega$ isolines and the corresponding streamlines.

Trapped wave trains several hundred kilometres in length arrive downstream of the mountain in the case of narrow- $(l=5 \mathrm{~km}$, Fig. 3$)$ and medium-width $(l=15 \mathrm{~km}$, Fig. 4$)$ orography. Beginning with $l \sim 20 \mathrm{~km}$, the trapped component declines and disappears completely at $l=25 \mathrm{~km}$ (Fig. 5). As we will see later, this decline is linked to the existence of short-wavelength resonance in the drag and stress spectra. Trapped waves are originated by wave reflection on the tropopause under conditions of small $N$ in the upper troposphere. Reflection is partial, and a fraction of the waves is refracted into the stratosphere in the form of upright secondary wave trains, as shown in Figs. 4 and 5.

To demonstrate the wind rotation and the critical level effects, Fig. 6 reproduces the modelling results for three different wind profiles: WM4 (non-rotating wind), WM5 (wind vanishing above $23.2 \mathrm{~km}$ altitude or $32 \mathrm{hPa}$ level), and WM6 (the complete nonsimplified wind profile). Their wave patterns look almost identical in the troposphere and lower stratosphere, as shown in Fig. 6(a). An essential wave breaking starts at $\sim$ $150 \mathrm{hPa}$ (14 km altitude). Above that level, waves in WM4 and WM6 still remain close (though presented is the wave pattern of WM4), but those in model WM5 decline with the full absorption of waves before the $32 \mathrm{hPa}$ level. Above approximately the $60 \mathrm{hPa}$ level, the wave field transforms to a quasi-regular, stationary, vertically oriented vortex field. For model WM4, the vortexes appear in Fig. 6(b) as horizontally stretched, vertically thin, closed streamline contours between 20 and $50 \mathrm{hPa}$ levels. The streamline pattern in the case of WM4 is shown with enhanced resolution in Figs. 6(b) and 6(c). As seen, the vortexes are formed in the shape of closed streamline contours. Indeed, the entire streamline contour pattern looks rather turbulent. Such turbulently fragmented wave field we will call the stratospheric quasi-turbulence (SQT). SQT originates from rapid decay of wind $u_{x}$ above the $100 \mathrm{hPa}$ level, which causes increasing wave phase delay with altitude and a strong tilt of $\omega$-isolines. This tilt itself is not sufficient for streamline fragmentation, as seen from Fig. 6(d), which shows a very strong negative tilt below the zero-wind level. In addition, the breaks in the wind profile (presented by the two horizontal lines in Figs. 6(b) and $6(\mathrm{c})$ ), are needed to provide the main fragmentation at passage through the lower 55 $h \mathrm{~Pa}$ level (the $24 \mathrm{~km}$ break of $U_{3}, U_{4}, U_{6}$ in Fig. 3) and further modification of SQT in passage through the upper $30 \mathrm{hPa}$ level (the $26.5 \mathrm{~km}$ break in Fig. 3).

The nearly identical behaviour of WM6 and WM4 shows that wind rotation has no significant effect on the wave pattern in two-dimensional wave generation experiments (this is not the case for stress spectra as we will see later). Concerning WM5 with critical levels (with vanishing for $p \leq 32 \mathrm{hPa}$ wind), its behaviour differs from that of the WM4 case at the very top, at $p<100 \mathrm{hPa}$. For WM5, all spectral waves are completely absorbed on the critical levels $p_{c}(k): k u\left[p_{c}(k)\right]=f$, which depend on $k$ being located slightly below 


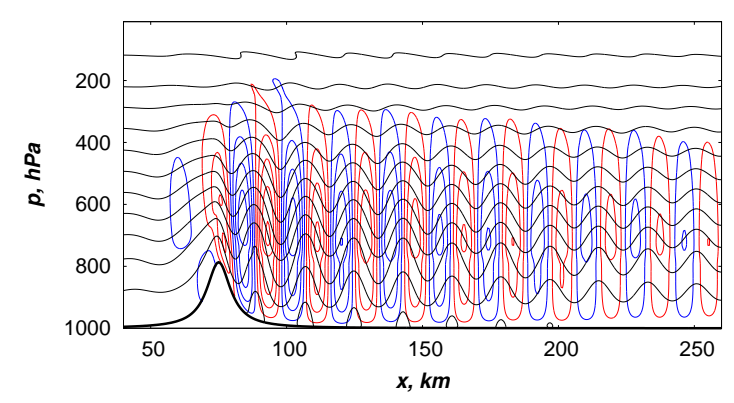

(a) WM4 through WM6; $\Delta \omega=20 \mathrm{~Pa} \mathrm{~s}^{-1}, \Delta \Psi^{v} / \bar{p}_{s}=2$ $\mathrm{ms}^{-1}$

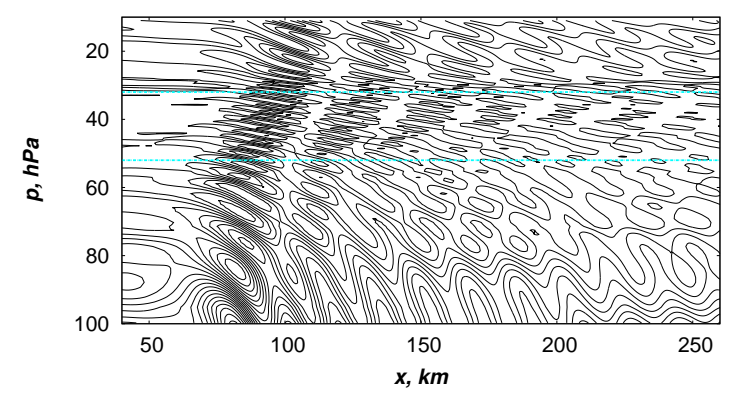

(c) WM4; $\Delta \Psi^{v} / \bar{p}_{s}=0.03 \mathrm{~m} \mathrm{~s}^{-1}$

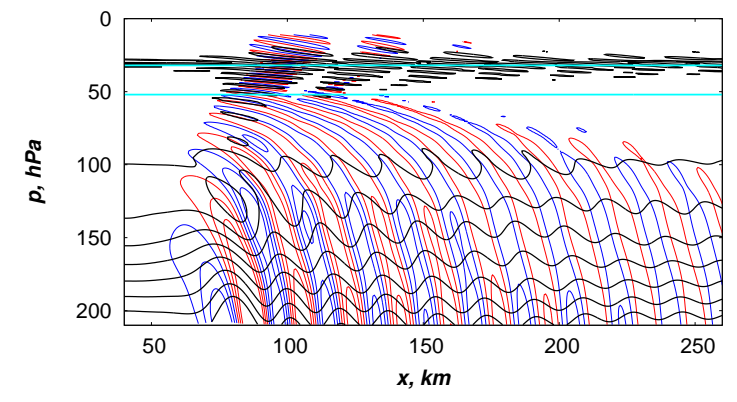

(b) WM4; $\Delta \omega=2 \mathrm{~Pa} \mathrm{~s}^{-1}, \Delta \Psi^{v} / \bar{p}_{s}=0.3 \mathrm{~m} \mathrm{~s}^{-1}$

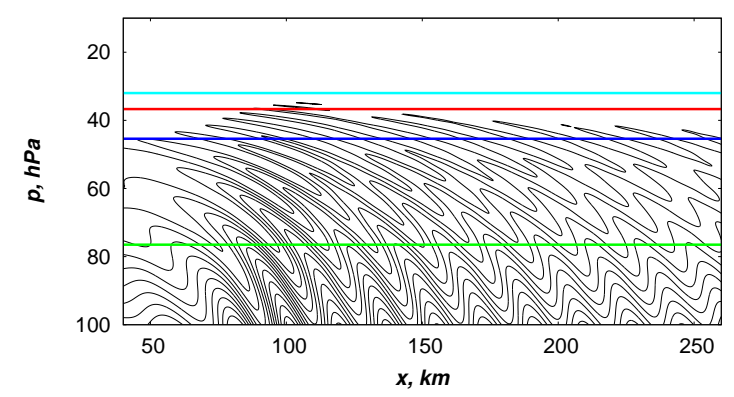

(d) WM5; $\Delta \Psi^{v} / \bar{p}_{s}=0.03 \mathrm{~m} \mathrm{~s}^{-1}$

Figure 6: Wave fields over a bell-shaped mountain with $l=5 \mathrm{~km}, h^{0}=2 \mathrm{~km}$ in the case of the wind models WM4, WM5 and WM6. In (a) and (b), the $\omega$-isolines are blue (rising mode) and red (descending mode); the streamlines are black in all panels. The two stripes of intensified vorticity in panels (b) and (c) are caused by the breaks in $u_{x}$ profile at $55 \mathrm{hPa}(z=23 \mathrm{~km})$ and $30 \mathrm{hPa}(z=26 \mathrm{~km})$, respectively. Horizontal lines in panel (d) mark velocity isolevels: $u_{x}=0$ (cyan), 1 (red), 2 (blue), and $5 \mathrm{~m} \mathrm{~s}^{-1}$ (green). For upper levels model WM6 is not shown because of behaving close to WM4.

the $p=32 h P a$ level but lie very close to it, because $f$ can be treated as a small parameter here.

\subsection{Surface drag}

Fig. 7 shows the modelling results of spectral drag amplitudes $A_{k}$ and $B_{k}$ as specified in (3.15a). To determine the wind effect, four profiles, WM1 to WM4, were applied, because cases WM5 and WM6 showed results very close to those of WM4. The longitudinal drag has a high narrow peak similar to line spectra in optics and acoustics. Such spectral drag resonance occurs because of tropospheric wave reflection on the tropopause and subsequent trapping in the troposphere, and is thus originated by the specific behaviour of $N$ in frontal conditions (small $N$ in the upper troposphere, followed by a large positive jump). The peak of $A_{k}$ is located at wavenumber $k_{r}$, coinciding well with the horizontal wavelength of the corresponding trapped wave train. The same is true for transverse drag $B_{k}$. It is mainly located near the resonance maximum $k_{r}$, where it has sharp high peaks with different signs on both sides of $k_{r}$. 

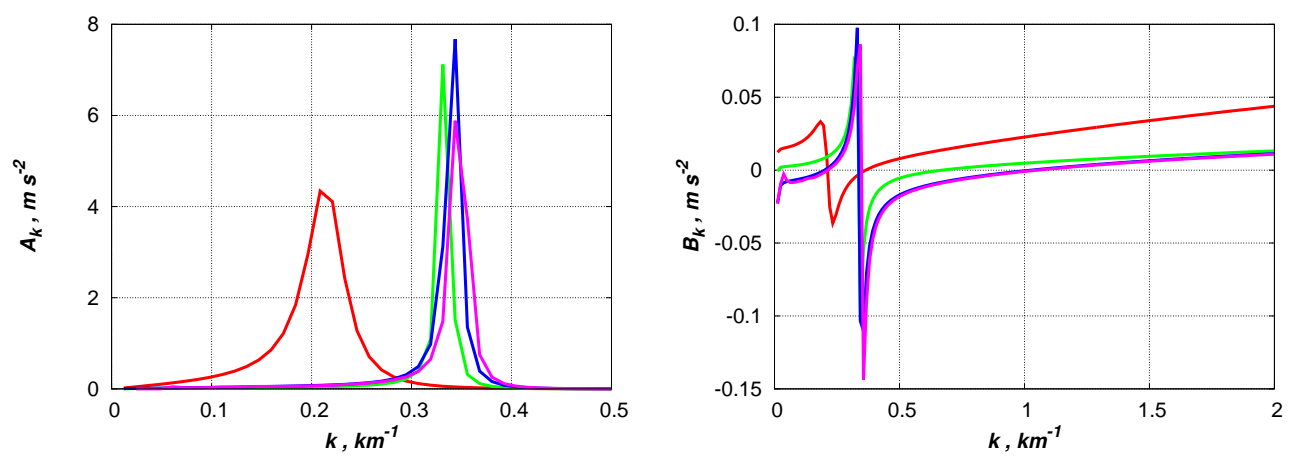

Figure 7: Longitudinal spectrum $A_{k}$ and transverse spectrum $B_{k}$ of surface drag according to (3.15a). Wind models: WM1 - red; WM2 - green; WM3 - blue; WM4 through WM6 - lilac.

Concerning moderation by wind, Fig. 7 shows that the strongest effect is exerted by the planetary boundary layer identified by the wind shear within it. If the boundary layer is absent (WM1 with constant wind $u_{x}$ throughout the entire atmosphere, including the boundary layer below $850 \mathrm{hPa}$ ), the resonant peaks of both longitudinal and transverse drag amplitudes are much lower and wider and the resonance maximum lies at $k_{r}=0.21$ $\mathrm{km}^{-1}$. That is, resonant waves without a boundary layer are much longer than those with a boundary layer. The shortening of horizontal wavelength by planetary boundary layer absorption is confirmed by earlier studies by [56,58] and by [59]. For WM2 through WM6 with boundary layer, the resonance maximum is located at $k_{r}=0.33 \mathrm{~km}^{-1}$, and the corresponding horizontal wavelength of the trapped waves $\lambda_{r}=2 \pi / k_{r}=19.0 \mathrm{~km}$ is in good agreement with that in Figs. 3 through 5. The drag contours of these models are extremely narrow and close to each other. At the same time, the wind shear and rotation in the stratosphere, the deep minimum at 22 through $26 \mathrm{~km}$ (WM6), or the complete wind nullification on the top (WM5) do not noticeably affect the resonance behaviour. Finally, it should be noted that the transverse surface drag remains modest in comparison with the longitudinal counterpart.

\subsection{Stress spectrum}

The stress amplitudes were computed according to the formulae in (3.15c). Fig. 8 shows 3D graphs of the longitudinal stress $\tau_{k}$ for WM4, WM5 and WM6. The longitudinal stress also becomes resonant, like the drag, in accordance with the relationships in (3.14), with a sharp crest at the same $k_{r}$.

In accordance with the longitudinal stress conservation for non-rotating wind as discussed above, $\tau_{k}$ remains constant with the altitude over the entire vertical extent of the atmosphere for WM1 through WM4 (the last case is shown in Fig. 8(a)), while for WM5 this happens below of the critical (wind vanishing) level at $23.2 \mathrm{~km}$ altitude. The critical level leads to a strong wave absorption and longitudinal stress abrupt fall to zero in a very narrow height interval immediately below the critical level, as seen in Fig. 8(c). 


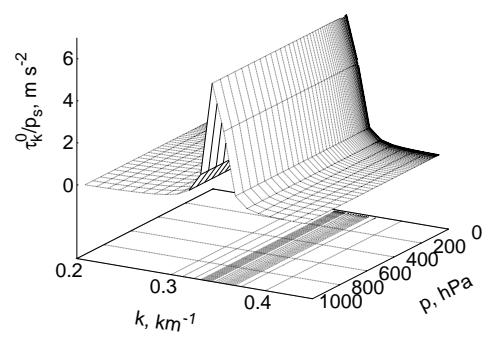

(a) WM4 (non-rotating wind)

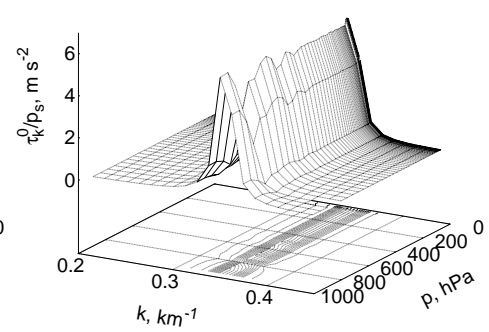

(b) WM6 (wind rotation included)

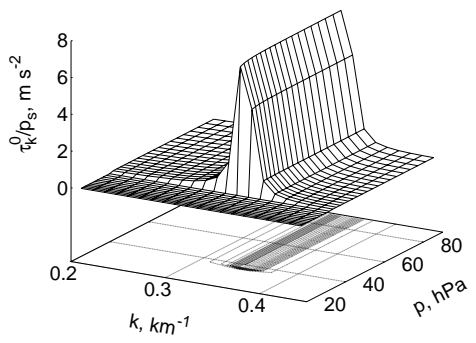

(c) WM5 (critical level, no rotation)

Figure 8: Spectra of longitudinal stress which demonstrate the wind rotation and critical level effect. The wind rotation causes an overall decrease in the resonance amplitude and substantial vertical variations in the lower troposphere, whereas the top wind nullification causes a noticeable resonance amplitude increase below the critical level.

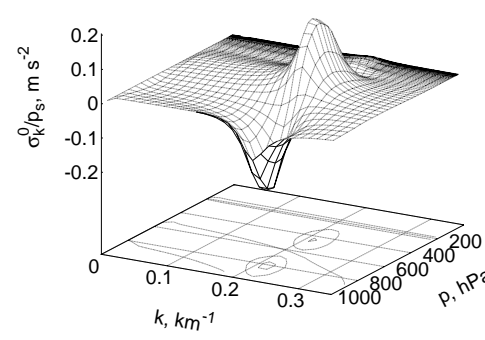

(a) WM1 (constant wind)
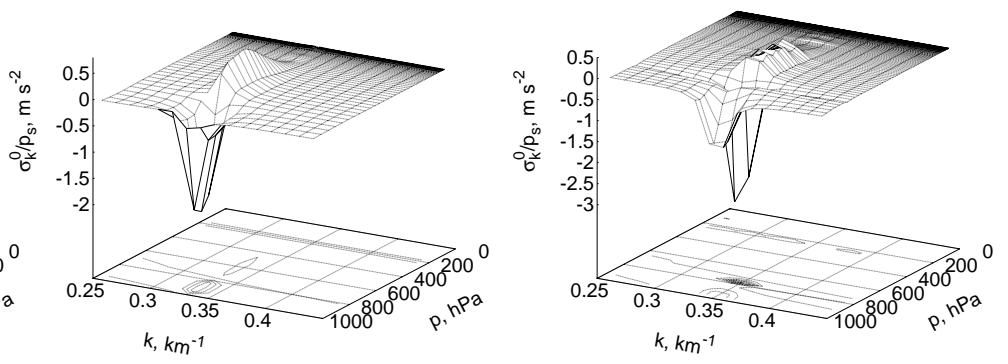

(b) WM2 (boundary layer included)

(c) WM6 (full wind with rotation)

Figure 9: Effect of wind shear and turn on the transverse stress conservation.

Though the wind rotation had no major effect on the wave pattern, the longitudinal stress becomes substantially convergent (Fig. 8(b)), which points to the substantial role of the third characteristic frequency $v_{k}^{3}$ in the wave equation for wind rotation conditions. In WM6, the longitudinal stress conservation is mainly violated where $v_{k}^{3}$ (i.e. wind rotation) becomes large, i.e. at the transition from the planetary boundary layer to the free atmosphere, with a strong minimum at $p \sim 800 \mathrm{hPa}$, and to a minor extent in the troposphere but not in the stratosphere. The distributions of transverse stress spectra are presented in Fig. 9 for WM1, WM2, and WM6 (WM3, WM4, and WM5 are similar to WM6 in this regard). As can be seen, the transverse stress also becomes resonant, with a sharp maximum coincident with the longitudinal drag and stress maximum $k_{r}$. In difference with the longitudinal counterpart, it is highly height-variable at all instances, though the variation amplitude is affected by the wind vertical variability. As an instance, the amplitude 
in the case of the constant wind in Fig. 9(a) is about one tenth of that with the boundary layer (Fig. 9(b)). The other essential quality of the transverse stress is that it becomes large at the transition from the planetary boundary layer to the troposphere and in the lower part of the troposphere, remaining small on the surface and completely vanishing at the tropopause and in the stratosphere. The relationship between transverse surface drag and stress in (3.14) is rather misleading in this respect, as the modest amplitudes of both $B_{k}$ and $\sigma_{k}^{0}\left(\bar{p}_{s}\right)$ give no hint that $\sigma_{k}^{0}$ will grow rapidly with the altitude.

\subsection{Wave drag}

The strong height dependence of the wave stress, evident from Figs. 8(b) and 9, should produce a noticeable wave drag, also. For closer investigation, in Figs. 10 and 11 the vertical distributions of the resonant, $k=k_{r}$, wave stress and wave drag are shown.

The resonant longitudinal wave stress in Fig. 10(a), corresponding to the rotating wind case WM6, reproduces the crest profile of Fig. 8(b). For comparison and emphasising of the great role of wind rotation on the longitudinal stress variability, the constant stress value for the non-rotating wind WM4 is shown with the red vertical line. Though the variability of the longitudinal stress in this example is large in the lower troposphere (comparable to the transverse stress variability in the same wind model), it almost vanishes (except of small vibrations) above $400 \mathrm{hPa}$, while stress tends to a constant value at the top, remaining finite and large everywhere. The total variation of $\tau_{k_{r}}^{0}(p) / \bar{p}_{s}$ in the whole vertical extent of the atmosphere consists $\sim 5.6-5.2=0.4 \mathrm{~ms}^{-2}$, which is $\sim 7 \%$ of its value on the surface. That is, major part of the longitudinal stress (which is the vertical flux of the horizontal momentum) remains non-absorbed at the reaching of top. The corresponding longitudinal drag in Fig. 10(b) exhibits coincidental large oscillations of the stress convergence and divergence, indicating that there exists substantial exchange of the longitudinal momentum between different layers in the troposphere. Small-amplitude drag protuberances in the stratosphere owe their genesis to the rapid vertical vibrations of the stress and are caused by the tiny variations in wind direction.

In Fig. 11(a), the height dependence of the resonant transverse stress $\sigma_{k_{r}}^{0}(p)$ is demonstrated for different wind models from Table 2. Graphs are shown for WM1 in red, WM2 in green, WM3 (close to WM4 and WM5 due to coincidental wind profiles below $7 \mathrm{~km}$ altitude) in dark blue, and WM6 in black. In the case of WM1 with uniform wind, the transverse stress has the smoothest profile and the lowest amplitude (approximately $10 \%$ of the longitudinal stress amplitude), with the maximum at the $450 \mathrm{hPa}$ level and a negative minimum at the $800 \mathrm{hPa}$ level. In case of WM2, the stress maximum descends to the 600 $h P a$ altitude and the minimum falls to the $900 \mathrm{hPa}$ level in the planetary boundary layer. In WM3 and WM6, the main drag minimum in the boundary layer is further lowered to the $970 \mathrm{hPa}$ level. The minimum of WM6, which is less prominent compared with that of WM3, is determined by near-surface wind rotation (directional shear) in WM6. The vertically narrow negative protrusive peak in the stress $\sigma_{k_{r}}^{0}$ at the $800 \mathrm{hPa}$ altitude in case of WM3 and WM6 is caused by a sharp break in the wind profile $u_{x}(p)$ at the transition 


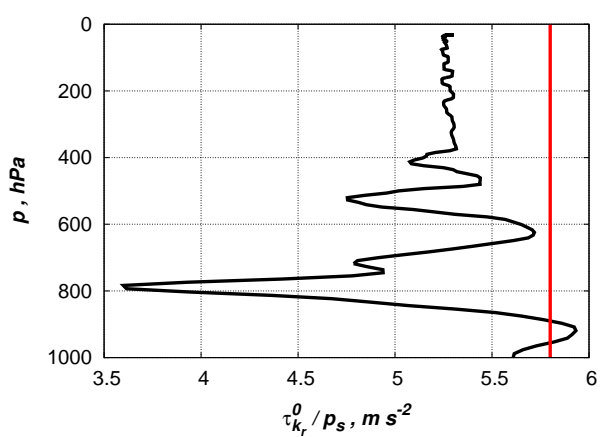

(a) WM6: longitudinal stress

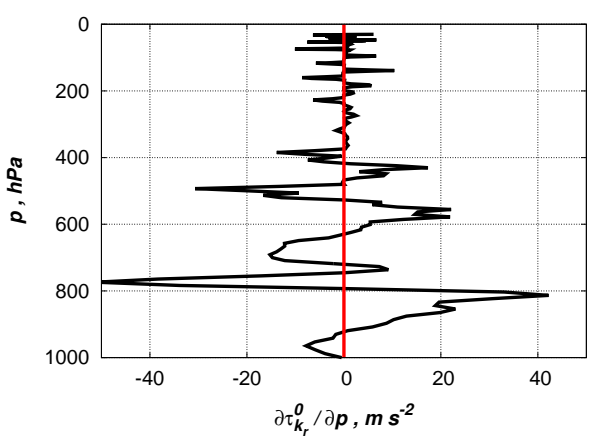

(b) WM6: longitudinal drag

Figure 10: Relative (divided by $\bar{p}_{S}$ ) longitudinal stress and longitudinal drag of WM6 at the resonance maximum $k_{r}$.

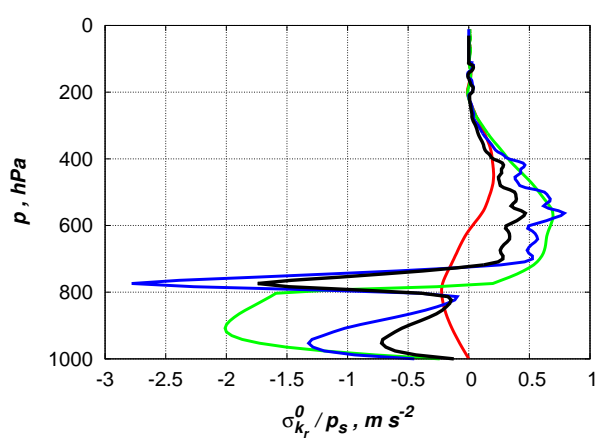

(a) WM1-WM3,WM6: transverse stress

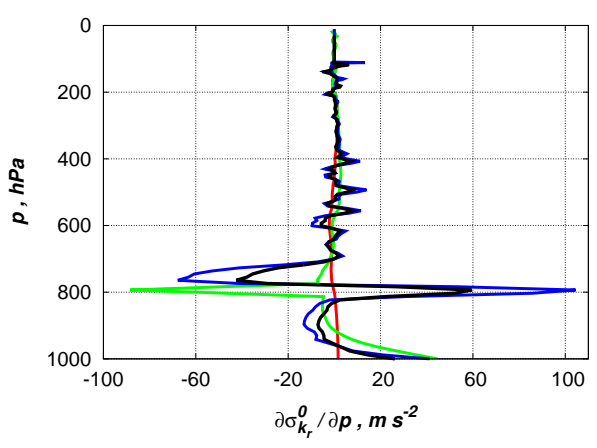

(b) WM1-WM3,WM6: transverse drag

Figure 11: Relative (divided by $\bar{p}_{s}$ ) transverse stress and the transverse drag at the resonance maximum $k_{r}$ for different wind models. Red - WM1; green - WM2; blue - WM3; black - WM6.

from the boundary layer to the free atmosphere. This peak originates from the second derivative of the wind $p^{2} \partial^{2} u_{x} / \partial p^{2}$ in the fourth characteristic frequency $v_{\left(k_{x}, 0\right)}^{4}$ in Scorer parameter $\Lambda$ (see (3.7)), as the third parameter $v_{\left(k_{x}, 0\right)}^{3}$ becomes zero for WM3. Despite of the large variability in details, all transverse stress profiles possess common large-scale quality: they all are zero on the surface and on the top, predominantly negative in the planetary boundary layer and in the lower troposphere below $700 \mathrm{hPa}(3 \mathrm{~km})$ level, and moderately large positive between 400 trough $700 \mathrm{hPa}(3$ through $7 \mathrm{~km})$ altitudes, with a steady decline to zero at the $200 \mathrm{hPa}$ (12 km tropopause) level.

The resonant transverse wave drag components $\partial \sigma_{k_{r}}^{0} / \partial p$ are shown in Fig. 11(b). This drag is rather modest in the model without boundary layer (WM1, red curve in Fig. 11(b)). The boundary layer inclusion in case of WM2 through WM6 causes a vast amplitude magnification ( $\sim 10$ to 20 times that of the homogeneous case WM1). Large outbreaks near $800 \mathrm{hPa}(\sim 2 \mathrm{~km})$ level in WM2, WM3 and WM6 correspond to the rapid changes of the stress $\sigma_{k_{r}}^{0}$. In general, the transverse drag is of the same amplitude with 
the longitudinal one in the case of the revolving wind WM6, but differently from the last one it is concentrated much lower, mainly in the first 3-km layer above the surface.

\section{Discussion}

The first two sections, Sections 2 and 3, were completely channelled to creation of a waveequation-based, linear, spectral stress and drag theory in pressure coordinates. Major effort was devoted to the stress conservation problem in Section 3.5. Application of the wave equation for conservation proof (first applied for conservation study in this investigation, to our best knowledge) enabled to classify the situations in which the longitudinal stress conserves and to make sure that the transverse stress never conserves exactly. The theoretical results about stress conservation were further confirmed by numerical modelling.

The longitudinal stress becomes conservative and produces no drag for non-rotating but other-way variable wind at an additional condition that the orography is two dimensional, so that the waves are likewise two-dimensional producing the exact vanish of characteristic frequencies $v_{\left(k_{x}, 0\right)}^{3}$ and $v_{\left(k_{x}, 0\right)}^{2}$ and approximate $v_{\left(k_{x}, 0\right)}^{1} \rightarrow 0$. This is the classical Eliassen-Palm zonal flow case. In all other occurrences (including both the rotating wind in $2 \mathrm{D}$ orography conditions and the non-rotating wind in the 3D orography conditions), the longitudinal stress remains convergent. However, as the following numerical treatment established, the conservation lack is not crucial for the longitudinal stress as there always remains a substantial non-absorbed momentum flux passing from the troposphere to stratosphere.

The transverse stress demonstrates height variability in all cases excluding the constant wind WM1, in which case it becomes rather small and the approximate conservation may be considered. That will mean, that for the complete stress vector $\gamma$ (without prior simplifications yielding the transverse stress exclusion), the only approximate conservation case is provided by the constant wind model. Even an inclusion of the boundary layer with wind shear (WM2 in Fig. 9) will destroy the conservation profoundly. In general, the transverse wave stress conversion presents an novel wave drag mechanism, which is different from the longitudinal case and which appears to be left out of consideration in former wave drag treatment and in available parameterisation schemes.

Though the surface drag (2.9d) was initially defined as the volume mean of the horizontal pressure and Coriolis forces, $((2.9 \mathrm{~b}),(2.9 \mathrm{c}))$, actually it represents the vertical average of highly altitude-dependent area-mean orographic drag forces $\left((2.6 b)^{\prime},(2.6 c)^{\prime}\right)$, which disappear on the trans-mountain levels. For numerical models which do resolve the planetary boundary layer (but still remain modest in the horizontal resolution capability, needing thus a surface drag parameterisation), the surface drag concept should be refined according to (2.9f)-(2.9h) and (2.9i). In the case of sufficiently high vertical resolution in the boundary layer, already a layer-wise vertical averaging of the orographic drag forces $\mathbf{a}$ and $\mathbf{b}$ could become meaningful. Anyway, the surface drag presents a low- 
level forcing which affects momentum on the inter-mountain levels $p>p_{m}$, including the planetary boundary layer. It can produce the (area-mean) blocking and be responsible for envelope-orography formation. Except of dependencies (3.14b) (which only indicate that both the surface drag and the wave stress are of the same wave origin), surface drag does not have much of a relationship with the upper-level momentum changes.

The trans-mountain level drag can be produced either by convergence of the wave stress or by the turbulent dissipation of waves at the breaking levels. Modelling reveals that both stress components can be highly variable with the altitude, depending on the mean wind shear and rotation with height, producing remarkable drag in the boundary layer and troposphere. Still, there exist substantial differences in their behaviour. As already discussed above, prime difference consists in the drag conservation sensitivity to the wind rotation. The longitudinal stress remains conservative and produces no drag, if the wind and orography both are two-dimensional and wind does not rotate; the transverse stress never conserves.

Another great difference between transverse and longitudinal stress and drag consists in their vertical distribution. The transverse stress is zero on the surface and disappears also on the top. Depending on the wind profile, it is predominantly negative with large local minimums in the planetary boundary layer and in the lower troposphere below $700 \mathrm{hPa}(3 \mathrm{~km})$ level and moderately large positive between 400 trough $700 \mathrm{hPa}$ ( 3 through $7 \mathrm{~km}$ ) altitudes with steady decline to zero at the $200 \mathrm{hPa}$ (12 km tropopause) level (Fig. 11(a)).

Differently from the transverse counterpart, the longitudinal stress is finite and large on the surface and remains such on the top even in the convergent case with the rotating wind WM6 (Fig. 10). Though its variability is of the same order with that of the transverse component, producing also drag of the same magnitude in the troposphere, a major part of the vertical flux of the horizontal momentum passes to the stratosphere and remains non-absorbed up to the top. Here is the point where the Eliassen and Palm hypothesis of the longitudinal stress absorption through wave breaking followed by turbulent dissipation must be attracted. According to this hypothesis, the drag is realised above the level where the waves break, e.g. in the upper troposphere and in the stratosphere. Up to the breaking level, the longitudinal stress is either conservative (idealised case of a nonrotating zonal flow and 2D orography) or if it is convergent, its decline up to the breaking level remains modest (differently from the transverse stress, which typically vanishes to that level) and will be absorbed in a turbulent layer above it. The critical levels present in this respect a special case of the complete stress absorption in a very tiny layer where the turbulent dissipation is not required (see Figs. 6(d), 8(c)). The typical wave breaking level is expected in the upper troposphere, though for high mountains, the breaking can start immediately above of mountain tops at the trans-mountain altitudes. In our frontal case with tropospheric trapped waves, the breaking starts in the stratosphere at $\sim 150$ $\mathrm{hPa}$ level (14 km altitude, see Fig. 6). The large breaking-level altitude can be explained here with the moderate mountain height in combination with the resonant trapped wave energy downstream transportation in the troposphere. If the breaking level dissipation 
is missing or not intense enough, the waves can rise higher into the stratosphere, to the levels above $75 \mathrm{hPa}(18 \mathrm{~km})$, where (friction- and dissipation-free) NWM predicts formation of SQT (stratospheric quasi-turbulence), a quasi-turbulent wave pattern in the shape of stream-line vortex system, as shown in Fig. 6. SQT is not a true turbulence as it is stationary, regular, and non-absorbing. It may be guessed that SQT will transform to real non-stationary turbulence due to nonlinear interactions between wave vortexes (left out in linear stationary model), causing their dissipation and further drag creation in the stratosphere. The sudden stratospheric warming events could also be explained with SQT-based wave energy dissipation at appropriate altitudes. However, such hypotheses require further study involving large eddy simulation methods or updating the wave equation with nonlinear advection-convection terms.

Summarising, the NWM presents a helpful tool for stress- and drag-related wave modelling in the arbitrary temperature, wind, and orography conditions. Its main advantages are very high spatial resolution, frictionless wave-propagation and application of non-reflective boundary conditions, permitting to resolve the finest details in the modelled wave fields throughout entire vertical extent of the atmosphere.

The further developments in NWM are mainly related with nonlinear effects, which include over-mountain bending of streamlines, planetary boundary layer interaction with orography, upper level wave breaking with turbulence generation, and possible nonstationary dissipation of SQT.

\section{Acknowledgments}

This work was supported by the Estonian Science Foundation (Grants 7478, 9134) and the Estonian Research Council (Targeted Financing Project SF0180038s08).

\section{References}

[1] T. N. Palmer, G. J. Shutts and R. Swinbank, Alleviation of a systematic westerly bias in general circulation and numerical weather prediction models through an orographic gravity wave drag parameterization, Q. J. R. Meteorol. Soc., 112 (1986), 1001-1039.

[2] N. A. McFarlane, The Effect of Orographically Excited Gravity Wave Drag on the General Circulation of the Lower Stratosphere and Troposphere, J. Atmos. Sci., 44 (1987), 1775-1800.

[3] P. G. Baines and T. N. Palmer, Rationale for a new physically based parametrization of subgrid scale orographic effects, ECMWF Tech. Memo, 169 (1990), ECMWF, Shinfield Park, Reading, Berkshire, U.K.

[4] B. Kirtman, A. Vernekar, D. DeWitt and J. Zhou, Impact of orographic gravity wave drag on extended-range forecasts with the COLA-GCM, Atmósfera, 6 (1993), 3-23.

[5] Y. J. Kim, S. D. Eckermann and H. Y. Chun, An Overview of the Past, Present and Future of Gravity-Wave Drag Parameterization for Numerical Climate and Weather Prediction Models, Atmosphere-Ocean, 41 (2003), 65-98.

[6] L. Rontu, A study on parametrization of orography-related momentum fluxes in a synopticscale NWP model, Tellus A, 58 (2006), 69-81. 
[7] H-Y. Chun, H-J. Choi and I-S. Song, Effects of Nonlinearity on Convectively Forced Internal Gravity Waves: Application to a Gravity Wave Drag Parameterization, J. Atmos. Sci., 65 (2008), 557-575.

[8] J. H. Richter, F. Sassi and R. R. Garcia, Toward a Physically Based Gravity Wave Source Parameterization in a General Circulation Model, J. Atmos. Sci., 67 (2010), 136-156.

[9] X. Zhu, J.-H. Yee, W. H. Swartz, E. R. Talaat and L. Coy,A Spectral Parameterization of Drag, Eddy Diffusion, and Wave Heating for a Three-Dimensional Flow Induced by Breaking Gravity Waves, J. Atmos. Sci., 67 (2010), 2520-2536.

[10] M. J. Alexander, M. Geller, C. McLandress, S. Polavarapu, P. Preusse, F. Sassi, K. Sato, S. Eckermann, M. Ern, A. Hertzog, Y. Kawatani, M. Pulido, T. A. Shaw, M. Sigmond, R. Vincent and S. Watanabe, Recent developments in gravity-wave effects in climate models and the global distribution of gravity-wave momentum flux from observations and models, Q. J. R. Meteorol. Soc., 136 (2010), 1103-1124.

[11] H-J. Choi and H-Y. Chun, Momentum Flux Spectrum of Convective Gravity Waves. Part I: An Update of a Parameterization Using Mesoscale Simulations, J. Atmos. Sci., 68 (2011), 739-759.

[12] J. S. Sawyer, The introduction of the effects of topography into methods of numerical forecasting, Q. J. R. Meteorol. Soc., 85 (1959), 31-43.

[13] R. B. Smith, The Influence of Mountains on the Atmosphere, Advances in Geophysics, 21 (1979), 87-230.

[14] R. B. Smith, Linear theory of stratified hydrostatic flow past an isolated mountain, Tellus, 32 (1980), 348-364.

[15] D. S. Phillips, Analytical Surface Pressure and Drag for Linear Hydrostatic Flow over ThreeDimensional Elliptical Mountains, J. Atmos. Sci., 41 (1984), 1073-1084.

[16] A. Eliassen and E. Palm, On the transfer of energy in stationary mountain waves, Geofysiske Publikasjoner, 22 (1961), 1-23.

[17] J. G. Charney and P. G. Drazin, Propagation of Planetary-Scale Disturbances from the Lower into the Upper Atmosphere, J. Geophys. Res., 66 (1961), 83-109

[18] D. G. Andrews and M. E. McIntyre, Planetary Waves in Horizontal and Vertical Shear: The Generalized Eliassen-Palm Relation and the Mean Zonal Acceleration, J. Atmos. Sci., 33 (1976), 2031-2048.

[19] F. P. Bretherton, Momentum transport by gravity waves, Q. J. R. Meteorol. Soc., 95 (1969), 213-243.

[20] W. R. Peltier and T. L. Clark, The Evolution and Stability of Finite-Amplitude Mountain Waves. Part II: Surface Wave Drag and Severe Downslope Windstorms, J. Atmos. Sci., 36 (1979), 1498-1529.

[21] D. R. Durran and J. B. Klemp, The Effects of Moisture on Trapped Mountain Lee Waves, J. Atmos. Sci., 39 (1982), 2490-2506.

[22] D. R. Durran and J. B. Klemp, A Compressible Model for the Simulation of Moist Mountain Waves, Mon. Weather Rev., 111 (1983), 2341-2361.

[23] R. T. Pierrehumbert, An essay on the parameterization of orographic gravity-wave drag, in Seminar/Workshop 1986: observation, theory, and modelling of orographic effects, 251-282. ECMWF, Shinfield Park, Reading, U.K., 1987.

[24] Y.-J. Kim and A. Arakawa, Improvement of Orographic Gravity Wave Parameterization Using a Mesoscale Gravity Wave Model, J. Atmos. Sci., 52 (1995), 1875-1902.

[25] D. R. Durran, Do Breaking Mountain Waves Decelerate the Local Mean Flow?, J. Atmos. Sci., 52 (1995), 4010-4032. 
[26] W. T. Welch, P. Smolarkiewicz, R. Rotunno and B. A. Boville, The Large-Scale Effects of Flow over Periodic Mesoscale Topography, J. Atmos. Sci., 58 (2001), 1477-1492.

[27] A. S. Broad, Momentum flux due to trapped lee waves forced by mountains, Q. J. R. Meteorol. Soc., 128 (2002), 2167-2173.

[28] J. D. Doyle and C. A. Reynolds, Implications of Regime Transitions for Mountain-WaveBreaking Predictability, Mon. Weather Rev., 136 (2008), 5211-5223.

[29] J. Lindeman, Z. Boybeyi, D. Broutman, Jun Ma, S. D. Eckermann and J. W. Rottman, Mesoscale Model Initialization of the Fourier Method for Mountain Waves, J. Atmos. Sci., 65 (2008), 2749-2756.

[30] J. B. Klemp and D. R. Lilly, The Dynamics of Wave-Induced Downslope Winds, J. Atmos. Sci., 32 (1975), 320-339.

[31] B. Grisogono, Dissipation of Wave Drag in the Atmospheric Boundary Layer, J. Atmos. Sci., 51 (1994), 1237-1243.

[32] M. Leutbecher, Surface Pressure Drag for Hydrostatic Two-Layer Flow over Axisymmetric Mountains, J. Atmos. Sci., 58 (2001), 797-807.

[33] J. R. Holton, J. H. Beres and X. Zhou, On the Vertical Scale of Gravity Waves Excited by Localized Thermal Forcing, J. Atmos. Sci., 59 (2002), 2019-2023.

[34] J. H. Beres, Gravity Wave Generation by a Three-Dimensional Thermal Forcing, J. Atmos. Sci., 61 (2004), 1805-1815.

[35] M. A. C. Teixeira and P. M. A. Miranda, The Effect of Wind Shear and Curvature on the Gravity Wave Drag Produced by a Ridge, J. Atmos. Sci., 61 (2004), 2638-2643.

[36] M. A. C. Teixeira and P. M. A. Miranda, A linear model of gravity wave drag for hydrostatic sheared flow over elliptical mountains, Q. J. R. Meteorol. Soc., 132 (2006), 2439-2458.

[37] M. A. C. Teixeira and P. M. A. Miranda, On the Momentum Fluxes Associated with Mountain Waves in Directionally Sheared Flows, J. Atmos. Sci., 66 (2009), 3419-3433.

[38] M. A. C. Teixeira, P. M. A. Miranda and J. L. Argaín, Mountain Waves in Two-Layer Sheared Flows: Critical-Level Effects, Wave Reflection, and Drag Enhancement, J. Atmos. Sci., 65 (2008), 1912-1926.

[39] R. Rõõm and M. Zirk, An Efficient Solution Method for the Buoyancy Wave Equation at Variable Wind and Temperature, Mon. Weather Rev., 135 (2007), 3633-3641.

[40] M. J. Miller, On the use of pressure as vertical co-ordinate in modelling convection, Q. J. R. Meteorol. Soc., 100 (1974), 155-162.

[41] M. J. Miller and R. P. Pearce, A three-dimensional primitive equation model of cumulonimbus convection, Q. J. R. Meteorol. Soc., 100 (1974), 133-154.

[42] R. Rõõm, A. Männik and A. Luhamaa, Non-hydrostatic semi-elastic hybrid-coordinate SISL extension of HIRLAM. Part I: numerical scheme, Tellus A, 59 (2007), 650-660.

[43] R. Rõõm, A. Männik and A. Luhamaa, Non-hydrostatic semi-elastic hybrid-coordinate SISL extension of HIRLAM. Part II: Numerical testing, Tellus A, 59 (2007), 661-673.

[44] R. Rõõm and A. Männik, Responses of Different Nonhydrostatic, Pressure-Coordinate Models to Orographic Forcing, J. Atmos. Sci., 56 (1999), 2553-2570.

[45] R. Rõõm, Nonhydrostatic adiabatic kernel for HIRLAM. Part I: Fundamentals of nonhydrostatic dynamics in pressure-related coordinates, HIRLAM Technical Report, 48 (2001), Available at http://hirlam.org/publications/TechReports/TR48.pdf .

[46] R. B. Smith, A Theory of Lee Cyclogenesis, J. Atmos. Sci., 41 (1984), 1159-1168.

[47] F. Lott, Alleviation of Stationary Biases in a GCM through a Mountain Drag Parameterization Scheme and a Simple Representation of Mountain Lift Forces, Mon. Weather Rev., 127 (1999), 788-801. 
[48] R. S. Scorer, Theory of waves in the lee of mountains, Q. J. R. Meteorol. Soc., 75 (1949), 41-66.

[49] A. S. Broad, Linear theory of momentum fluxes in 3-D flows with turning of the mean wind with height, Q. J. R. Meteorol. Soc., 121 (1995), 1891-1902.

[50] D. R. Durran, Numerical Methods for Wave Equations in Geophysical Fluid Dynamics, Springer (1998), $465 \mathrm{p}$.

[51] R. B. Smith, Further Development of a Theory of Lee Cyclogenesis, J. Atmos. Sci., 43 (1986), 1582-1602.

[52] Undén, P., L. Rontu, H. Järvinen, P. Lynch, J. Calvo, G. Cats, J. Cuxart, K. Eerola, C. Fortelius, J. A. Garcia-Moya, C. Jones, G. Lenderlink, A. McDonald, R. McGrath, B. Navascues, N. W. Nielsen, V. Ødegaard, E. Rodriguez, M. Rummukainen, R. Rõõm, K. Sattler, B. H. Sass, H. Savijärvi, B. W. Schreur, R. Sigg, H. The and A. Tijm, HIRLAM-5 Scientific Documentation, December 2002. Swedish Meteorological and Hydrological Institute, Norrköping, Sweden. 2002.

[53] L. B. Nance and D. R. Durran, A Modeling Study of Nonstationary Trapped Mountain Lee Waves. Part I: Mean-Flow Variability, J. Atmos. Sci., 54 (1997), 2275-2291.

[54] L. B. Nance and D. R. Durran, A Modeling Study of Nonstationary Trapped Mountain Lee Waves. Part II: Nonlinearity, J. Atmos. Sci., 55 (1998), 1429-1445.

[55] , S. B. Vosper, P. F. Sheridan and A. R. Brown, Flow separation and rotor formation beneath two-dimensional trapped lee waves, Q. J. R. Meteorol. Soc., 132 (2006), 2415-2438.

[56] Q. Jiang, J. D. Doyle and R. B. Smith, Interaction between Trapped Waves and Boundary Layers, J. Atmos. Sci., 63 (2006), 617-633.

[57] V. Grubišić and I. Stiperski, Lee-Wave Resonances over Double Bell-Shaped Obstacles, J. Atmos. Sci., 66 (2009), 1205-1228.

[58] Q. Jiang, R. B. Smith and J. D. Doyle, Impact of the Atmospheric Boundary Layer on Mountain Waves, J. Atmos. Sci., 65 (2008), 592-608.

[59] I. Stiperski and V. Grubisicic, Trapped Lee Wave Interference in the Presence of Surface Friction, J. Atmos. Sci., 68 (2011), 918-939. 